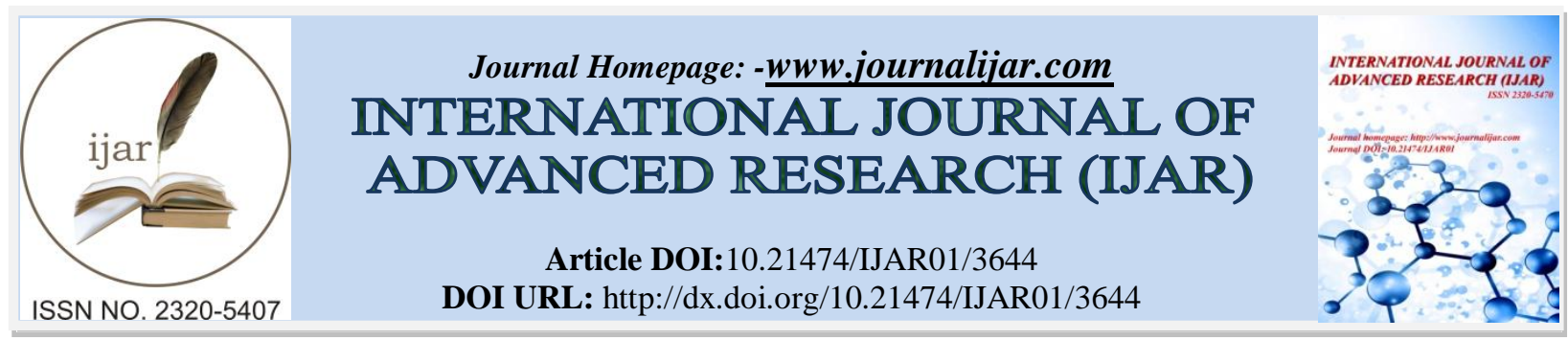

RESEARCH ARTICLE

\title{
A FOOD CHAIN STRUCTURES AND DYNAMICS ACROSS A REFUGE STAGE- STRUCTURE PREY- PREDATOR MODEL.
}

Azhar Abbas Majeed and Sabah Ali Rahi.

Department of Mathematics, College of Science, University of Baghdad, Baghdad, Iraq.

\section{Manuscript Info}

Manuscript History

Received: 09 January 2017

Final Accepted: 03 February 2017

Published: March 2017

food chain, refuge, stage- structure, stability analysis.
Key words:functional response, Lyapunov function,

\begin{abstract}
In the present paper the structures and dynamics of a food chain preypredator model are proposed and studied. The two stages and refuge in prey and two predators (top and mid) are considered. Different types of functional responses have been proposed. The conditions, which guarantee the existence of equilibrium points, have been investigated. Uniqueness and boundedness of the solution of the system are proven. The local and global dynamical behaviors are discussed and analyzed. Finally, numerical simulations are carried out not only to confirm the theoretical results obtained, but also to show the effects of the refuge and the variation of each parameter on our proposed system.
\end{abstract}

Copy Right, IJAR, 2017,. All rights reserved.

\section{Introduction:-}

The first pattern formation of predator-prey model was introduced in 1927 by the well- known Lotka[15] and Volterra[28] which starting from a simple and classical supposition, more complexity but factual predator-prey models have been constructed by mathematicians and ecologists. In 1992, Berryman [6] considered that the dynamic interaction between predators and preys has long taken and go on to be one of the most important and central subjects which play a great role in each of the mathematical ecology, natural, social, and technological sciences, especially in the research on biology and ecology [5, 17, 26, 27].

Many researchers working in these fields paying a great amount of attention to improve and generalize the pattern formation of prey- predator models to more involvement and realistic system, and merging this advantage into many applications that give the nature of existence and global asymptotic stability of predator-prey models a great interest.

On the other hand, the dynamical behavior of prey-predator models has been investigated by a lot of articles and peppers which results a significant expansion in the structural of the prey - predator models to involve multispecies; different functional responses, several types of food chain, refuge, harvesting, stage-structure, several types of diseases...etc.

Lately, Samantha[25] studied the existence and global asymptotic stability of a delay predator-prey model with disease in the prey, Paletal.[20-24]proposed prey-predator harvesting model under impreciseness by considering biological parameters as interval number, Naji and Mustafa [19] studied the dynamics of an eco-epidemiological model with nonlinear incidence rate. Majeed and Shawka studied prey-predator model involving SI and SIS infectious disease in prey population and the disease transmitted within the same species by contact and external

Corresponding Author:-Azhar Abbas Majeed.

Address:-Department of Mathematics, College of Science, University of Baghdad, Baghdad, Iraq. 
source [4]. A lot of researches efforts have been made to investigating the interaction and coexistence mechanism of the harvested prey-predator.

In fact, the influence of refuge, stage-structure and the multiplicity of functional responses in the prey-predator ecosystem are the most important topics of interest. In recent years, stage structure models have been studies widely by a lot of researchers, Aiello and Freedman (1990) studied the single species model with stage structure [1, 2], several researchers studied prey-predator model with different functional responses. The prey-predator models with prey refuge have been investigated by $\operatorname{Kar}[14]$. Maetal. [16]studied the effects of prey refuges on a prey-predator model with a class of function responses. Chenetal.[8] discussedaprey-predator model with Holling type II functiona lresponse in corporating a constant prey refuge.

Moreover, someoftheexperimentalandtheoreticalworkhavestudiedtheeffectsofpreyrefuges and drawn a conclusion thatthepreyrefuges have a positively effectonthestabilityof the consideredinteractions,andprey extinction canbeconserved by theadditionofrefuges[9, 11, 12, 13, 17], Kadhim, Majeed and Naji [29] studied the stability analysis of food web stage structured prey-predator model with refuge involving Lotka-Volterra type of function response, Ali and Majeed discussed stability analysis of a food chain stage structured prey-predator model incorporating a preyrefuge and two types of functional responses such that the mid-predator consumed the mature prey individual only according to the Lotka-Voltera type of functional response and the top predator consumed the mid- predator individual only according to Holling type-II functional response.

In spite of the effect of prey refuges on the dynamical behavior of the system is very difficult in the reality, but it has been considered and analysis in this paper.

In this paper, a complicated four species food chain model with two functional responses has been proposed and analyzed. Two such stages (mature and immature) with refuge in the prey and two predators (top and mid) are also considered.

It is well known that the logistic function represents a mutualism interaction between the mature and immature prey, and due to the short of resources the typeIIfunctionalresponse is considered to describe the nature of the interaction between the mid predator and immature prey, while Lotka-Volterra type of functionalresponse considered due to unlimited resources and the linearly relationship between the mid predator and mature prey.

Furthermore, there is not any relationship between the top predator and the two stages of prey, while according to a food chain the interaction between the two predators has been considered by Lotka-Volterra type of functionalresponse.

Actually, due to the complexity of interactions in the proposed model especially among the top predator and the two stages of prey and the nature of interaction between the mid predator and the immature prey it was not easy to find an example identical of this model in the nature, but in any case is not impossible, so after more efforts and with the help of the biologist, we could find an example across two different environments represented by the aquatic and terrestrial environment such that the two aquatic species Blue Shark (Prionaceglauca) and Wels Catfishrepresent the top and mid predators respectively, and the terrestrial species pigeons (doves ) represent the two stages of prey.

Finally, the behavior of the catfish when hunting pigeons is highlighted in the study, published on Dec. 5. The study says: "Among a total of 45 beaching behaviors observed and filmed, $28 \%$ were successful in bird capture... Since this extreme behavior has not been reported in the native range of the species, our results suggest that some individuals in introduced predator populations may adapt their behavior to forage on novel prey in new environments, leading to behavioral and trophic specialization to actively cross the water-land interface." $[10,7]$.

\section{The mathematical model:-}

Consider the food chain model consisting of mid and top- predators, stage-structure prey in which the prey species growth logistically in the absence of predation, while the predators decay exponentially in the absence of prey species. It is assumed that the prey population divides into two compartments: immature prey population $X_{1}(\mathrm{t})$ that represents the population size at time $\mathrm{t}$ and mature prey population $X_{2}(\mathrm{t})$ which denotes to population size at time $\mathrm{t}$. Furthermore the population size of the mid- predator at time $\mathrm{t}$ is denoted by $Y_{1}(\mathrm{t})$, while $Y_{2}(\mathrm{t})$ represents the population size of top predator at time $t$. 
Now in order to formulate the dynamics of such system the following assumptions are considered:

1. The immature prey depends completely in its feeding on the mature prey that growth logistically with intrinsic growth rater $>0$ and carrying capacity $k>0$. The immature prey individuals grown up and become mature prey individuals with grown up rate $s>0$. However the immature and mature prey facing death with natural death rate $d_{1}>0$ and $d_{2}>0$ respectively.

2. There is type of protection of the prey species from facing predation by the mid- predator with refuge rate constant $m \in(0,1)$.

3. The mid-predator consumed the immature prey individual according to Holling type-II functional response with predation rate $a_{1}>0$ and half saturation constant $b>0$. And consumed the mature prey individual according to theLotka-Voltera type of functional response with predation rate $a_{2}>0$ and contribute a portion of such food with conversion rates $0<e_{1}<1$ and $0<e_{2}<1$ respectively. Moreover, the top predator consumed the mid-predator individual only according to the Lotka-Voltera type of functional response with predation rate $a_{3}>0$ and contributes a portion of such food with conversion rate $0<e_{3}<1$.

Finally, in the absence of food the mid and top predators facing death with natural death $\operatorname{rated}_{3}>0$ andd $_{4}>0$ respectively.

Therefore the dynamics of the above proposed model can be represented by the following set of first order nonlinear differential equations.

$\frac{d X_{1}}{d T}=r X_{2}\left(1-\frac{X_{2}}{k}\right)-\frac{a_{1}(1-m) X_{1}}{b+X_{1}} Y_{1}-s X_{1}-d_{1} X_{1}$

$\frac{d X_{2}}{d T}=s X_{1}-a_{2}(1-m) X_{2} Y_{1}-d_{2} X_{2}$

$\frac{d Y_{1}}{d T}=\frac{e_{1} a_{1}(1-m) X_{1}}{b+X_{1}} Y_{1}+e_{2} a_{2}(1-m) X_{2} Y_{1}-a_{3} Y_{1} Y_{2}-d_{2} Y_{1}$

$\frac{d Y_{2}}{d T}=e_{3} a_{3} Y_{1} Y_{2}-d_{4} Y_{2}$.

With initial conditions $X_{i}(0) \geq 0$ and $Y_{i}(0) \geq 0, i=1,2$.

Not that the above proposed model has fourteen parameters in all which make the analysis difficult. So in order to simplify the system, the number of parameters is reduced by using the following dimensionless variables and parameters:

$t=r \mathrm{~T}, u_{1}=\frac{\mathrm{b}}{\mathrm{k}}, u_{2}=\frac{\mathrm{s}}{\mathrm{r}}, u_{3}=\frac{\mathrm{d}_{1}}{\mathrm{r}}, u_{4}=\frac{\mathrm{a}_{2} \mathrm{k}}{\mathrm{a}_{1}}, u_{5}=\frac{\mathrm{d}_{2}}{\mathrm{r}}, a_{6}=\frac{e_{1} a_{1}}{r}, u_{7}=\frac{e_{2} a_{2} k}{r}$,

$u_{8}=\frac{d_{3}}{r}, u_{9}=\frac{e_{3} a_{3} k}{r}, u_{10}=\frac{d_{4}}{r}, x=\frac{X_{1}}{k}, y=\frac{X_{2}}{k}, \quad z=\frac{a_{1} Y_{1}}{r k}, w=\frac{a_{3} Y_{2}}{r}$.

Then the non-dimensional form of system (1) can be written as:

$\frac{d x}{d t}=x\left[\frac{y(1-y)}{x}-\frac{(1-m) z}{u_{1}+x}-\left(u_{2}+u_{3}\right)\right]=f_{1}(x, y, z, w)$

$\frac{d y}{d t}=y\left[\frac{u_{2} x}{y}-u_{4}(1-m) z-u_{5}\right]=f_{2}(x, y, z, w)$

$\frac{d z}{d t}=z\left[\frac{u_{6}(1-m) x}{u_{1}+x}+u_{7}(1-m) y-w-u_{8}\right]=f_{3}(x, y, z, w)$

$\frac{d w}{d t}=w\left[u_{9} z-u_{10}\right]=f_{4}(x, y, z, w)$.

With $x(0) \geq 0, y(0) \geq 0, z(0) \geq 0$ andw $(0) \geq 0$.

It is observed that the number of parameters have been reduced from fourteen in the system( 1 )to eleven in the $\operatorname{system}(2)$.

Obviously the interaction functions of the system ( 2 ) are continuous and have continuous partial derivatives on the following positive four dimensional space.

$R_{+}^{4}=\left\{(x, y, z, w) \in R^{4}: x(0) \geq 0, y(0) \geq 0, z(0) \geq 0, w(0) \geq 0\right\}$. 
Therefore these functions are Lipschitzian on $R_{+}^{4}$, and hence the solution of the system( 2 )exists and is unique. Further, all the solutions of system( 2 ) with non-negative initial conditions are uniformly bounded as shown in the following theorem.

Theorem (1):All the solutions of system ( 2 ) which initiate in $R_{+}^{4}$ are uniformly bounded.

Proof: Let $(x(t), y(t), z(t), w(t))$ be any solution of the system (2) with non-negative initial condition $\left(x_{0}, y_{0}, z_{0}, w_{0}\right) \in R_{+}^{4}$.

According to the first equation of system ( 2 ) we have

$\frac{d x}{d t}=y(1-y)-\frac{(1-m) x z}{u_{1}+x}-\left(u_{2}+u_{3}\right) x$.

So, $\frac{d x}{d t} \leq \frac{1}{4}-\left(u_{2}+u_{3}\right) x$.

Now, by using the comparison theorem on the above differential inequality with the initialpoint $x(0)=x_{0}$, weget: $x(t) \leq \frac{1}{4\left(u_{2}+u_{3}\right)}+\left(x_{0}-\frac{1}{4\left(u_{2}+u_{3}\right)}\right) e^{-\left(u_{2}+u_{3}\right) t}$.

Thus, $\lim _{t \rightarrow \infty} x(t) \leq \frac{1}{4\left(u_{2}+u_{3}\right)}$, and hence $\sup x(t) \leq \frac{1}{4\left(u_{2}+u_{3}\right)}, \quad \forall t>0$.

Now define the function: $N(t)=x(t)+y(t)+z(t)+w(t)$, and then taken the time derivative of $N(t)$ along the solution of the

$$
\frac{d N}{d t}=y-y^{2}-\left(1-u_{6}\right) \frac{(1-m) x z}{u_{1}+x}-\left(u_{4}-u_{7}\right) y z-\left(1-u_{9}\right) z w-u_{3} x-u_{5} y-u_{8} z-u_{10} w .
$$

So, due to the fact that the conversion rate constant from prey population to predator population cannot exceeding the maximum predation rate constant from predator population to prey population, hence from the biological point of view, always $u_{6}<1, u_{9}<1$ and $u_{7}<u_{4}$, we get,

$$
\frac{d N}{d t} \leq y-s N, \quad \text { where } \quad \mathrm{s}=\min \left\{u_{3}, u_{5}, u_{8}, u_{10}\right\}, \quad \text { and hence, } \quad \frac{d N}{d t} \leq \frac{u_{2}}{4 a_{5}\left(u_{2}+u_{3}\right)}-s N .
$$

Then, $\frac{d N}{d t}+s N \leq G, \quad$ where $G=\frac{u_{2}}{4 a_{5}\left(u_{2}+u_{3}\right)}$.

Again by solving this differential inequality for the initial value $N(0)=N_{0}$, we get:

$N(t) \leq \frac{G}{s}+\left(N_{0}-\frac{G}{s}\right) e^{-s t}$. Then, $\lim _{t \rightarrow \infty} N(t) \leq \frac{G}{s}$. So , $0 \leq N(t) \leq \frac{G}{s}, \forall t>0$,

hence all the solutions of system ( 2 ) are uniformly bounded and the proof is complet.

\section{The existence of equilibrium points:-}

In this section, the existence of all possible equilibrium points of system ( 2 )is discussed. It is observed that, system ( 2 ) has at most four equilibrium points, which are mentioned in the following:

OThe equilibrium point $E_{0}=(0,0,0,0)$, which known as vanishing point is always exists.

OThe free predators' equilibrium point $E_{1}=(\bar{x}, \bar{y}, 0,0) \mathrm{t}$, where:

$\bar{y}=1-u_{5}\left(\frac{u_{2}+u_{3}}{u_{2}}\right)$, and $\bar{x}=\frac{u_{5}}{u_{2}}\left(1-u_{5}\left(\frac{u_{2}+u_{3}}{u_{2}}\right)\right.$, exists uniquely in Int. $R_{+}^{2}$ (Interior of $\left.R_{+}^{2}\right)$ of $x y-p l a n e$ if the necessary condition holds:

$$
u_{5}<\frac{u_{2}}{u_{2}+u_{3}} .
$$

OThe free top predator' equilibrium point $E_{2}=(\hat{x}, \hat{y}, \hat{z}, 0)$, where:

$$
\hat{z}=\frac{u_{2} x-u_{5} y}{u_{4}(1-m) y}
$$

And,

$$
\hat{y}=\frac{u_{8}\left(u_{1}+x\right)-u_{6}(1-m) x}{u_{7}(1-m)\left(u_{1}+x\right)} .
$$

While, $\hat{x}$ is a positive root of the following fourth order polynomial:

$$
f(x)=\gamma_{1} x^{4}+\gamma_{2} x^{3}+\gamma_{3} x^{2}+\gamma_{4} x+\gamma_{5}=0,
$$

where:

$$
\begin{aligned}
& \gamma_{1}=u_{7}^{2}(1-m)^{3}\left[u_{4}\left(u_{2}+u_{3}\right)\left(u_{6}(1-m)-u_{8}\right)-u_{2} u_{7}(1-m)\right] \\
& \gamma_{2}=-u_{4}(1-m)\left(u_{6}(1-m)-u_{8}\right)\left[u_{7}(1-m)+\left(u_{6}(1-m)-u_{8}\right)^{2}\right] \\
& -u_{7}^{2}(1-m)^{3}\left[u_{5}\left(u_{6}(1-m)-u_{8}\right)+u_{1} u_{4} u_{8}\left(u_{2}+u_{3}\right)\right]-2 u_{1} u_{7}^{2}(1-m)^{3}\left[u_{2} u_{7}(1-m)-u_{4}\left(u_{6}(1-m)-u_{8}\right)\right] .
\end{aligned}
$$


$\gamma_{3}=u_{1} u_{4} u_{8}(1-m)\left(u_{6}(1-m)-u_{8}\right)\left[3\left(u_{6}(1-m)-u_{8}\right)-2 u_{7}(1-m)\right]+u_{1} u_{4} u_{7}(1-m)^{2}\left(u_{6}(-m)-u_{8}\right)^{2}$ $-u_{1}^{2} u_{4} u_{7}^{2}\left(u_{2}+u_{3}\right)(1-m)^{3}\left(3 u_{8}-u_{6}(1-m)\right)+u_{1} u_{7}^{2}(1-m)^{3}\left[u_{5} u_{8}-u_{1} u_{2} u_{7}(1-m)\right]$

$-u_{1} u_{5} u_{7}^{2}(1-m)^{3}\left(u_{6}(1-m)-u_{8}\right)$.

$\gamma_{4}=u_{1}^{2} u_{7} u_{8}(1-m)^{2}\left[u_{4} u_{8}+u_{5} u_{7}(1-m)\right]-u_{1}^{2} u_{4} u_{8}(1-m)\left(u_{6}(1-m)-u_{8}\right)\left[3 u_{8}+2 u_{7}(1-m)\right]$

$-u_{1}^{3} u_{7}^{2} u_{4} u_{8}(1-m)^{3}\left(u_{2}+u_{3}\right)$.

$\gamma_{5}=u_{1}^{3} u_{4} u_{7} u_{8}^{2}(1-m)^{2}+u_{1}^{3} u_{4} u_{8}^{3}(1-m)>0$.

Clearly, by using Descartes' rule of sign Eq. $(4 c)$ hasa unique positive root, denoted by $\hat{x}$, if

$$
\gamma_{i}<0, \quad i=1,2,3 \text { and }\left(\gamma_{4}<0 \text { or } \gamma_{4}>0\right) \text {. }
$$

Therefore, the free top predator' equilibrium point $E_{2}=(\hat{x}, \hat{y}, \hat{z}, 0)$ exists uniquely in the interior $R_{+}^{3}$ of $x y z-$ space if the following conditions hold:

$$
\begin{aligned}
& \frac{u_{6}(1-m)}{3}<u_{8}<u_{6}(1-m) \text {. } \\
& u_{2}>\max \left\{\frac{u_{5} \hat{y}}{\hat{x}}, \frac{u_{5} u_{8}}{u_{1} u_{7}(1-m)}\right\} \text {. } \\
& \left(u_{6}(1-m)-u_{8}\right)<\min \frac{u_{\operatorname{mg}} \frac{u_{1}}{2} u_{8}}{\hat{x}}, \frac{2 u_{7}(1-m)}{3}, \frac{u_{2} u_{7}(1-m)}{u_{4}}, \frac{u_{2} u_{7}(1-m)}{u_{4}\left(u_{2}+u_{3}\right)} \text {, } \\
& \left.u_{1} u_{7}\left(u_{2}+u_{3}\right)(1-m)\left(3 u_{8}-u_{6}(1-m)\right)\right\} \text {. }
\end{aligned}
$$

OFinally, the positive (coexistence) equilibrium point $E_{3}\left(x^{*}, y^{*}, z^{*}, w^{*}\right)$, where:

$$
\begin{aligned}
& z^{*}=\frac{u_{10}}{u_{9}} . \\
& w^{*}=\frac{u_{6}(1-m) x+u_{7}(1-m)\left(u_{1}+x\right) y-u_{8}\left(u_{1}+x\right)}{u_{1}+x} . \\
& x^{*}=\frac{\left(u_{4} u_{10}(1-m)+u_{5} u_{9}\right) y}{u_{2} u_{9}} .
\end{aligned}
$$

While, $y^{*}$ is a positive root of the following polynomial:

where:

$$
f(y)=y\left(\delta_{1} y^{2}+\delta_{2} y+\delta_{3}\right)=0
$$

$\delta_{1}=-u_{2} u_{9}\left(u_{4} u_{10}(1-m)+u_{5} u_{9}\right)<0$.

$\delta_{2}=u_{2} u_{9}\left(u_{4} u_{10}(1-m)+u_{5} u_{9}\right)-u_{1} u_{2}^{2} u_{9}^{2}-\left(u_{2}+u_{3}\right)\left(u_{4}(1-m)+u_{5} u_{9}\right)^{2}$.

$\delta_{3}=u_{2}\left[u_{1} u_{2} u_{9}^{2}-\left(u_{4} u_{10}(1-m)+u_{5} u_{9}\right)\left(u_{10}(1-m)+u_{9}\left(u_{2}+u_{3}\right)\right)\right]$.

Straightforward computation shows that the quadratic eq. $(5 d)$, has a unique positive root, denoted by $y^{*}$, if and only if the following condition holds:

$$
u_{1}>\frac{\left(u_{4} u_{10}(1-m)+u_{5} u_{9}\right)\left(u_{10}(1-m)+u_{9}\left(u_{2}+u_{3}\right)\right)}{u_{2} u_{9}^{2}} .
$$

Therefore, the positive (coexistence) equilibrium point $E_{3}\left(x^{*}, y^{*}, z^{*}, w^{*}\right)$ exists uniquely in the interior $R_{+}^{4}$ if in addition to the condition ( 5 e) the following condition holds:

$$
y^{*}>\frac{u_{8}}{u_{7}(1-m)} \text {. }
$$

\section{The local stability analysis of system(2)}

In this section, the local stability analysis of system( 2 )around each of the above equilibrium points is discussed through computing the Jacobian matrix $J(x, y, z, w)$ of system( 2 )at each of them which is given by:

$$
\begin{aligned}
& J=\left[a_{i j}\right]_{4 \times 4}, \text { where } \\
& a_{11}=-\frac{u_{1}(1-m) z}{\left(u_{1}+x\right)^{2}}-\left(u_{2}+u_{3}\right), \quad a_{12}=1-2 y, \quad a_{13}=-\frac{(1-m) x}{u_{1}+x}, \quad a_{14}=0, \quad a_{21}=u_{2}, \\
& a_{22}=-u_{4}(1-m) z-u_{5}, a_{23}-u_{4}(1-m) y, a_{24}=0, \quad a_{31}=\frac{u_{1} u_{6}(1-m) z}{\left(u_{1}+x\right)^{2}}, \quad a_{32}=u_{7}(1-m) z, \\
& a_{33}=\frac{u_{6}(1-m) x}{u_{1}+x}+u_{7}(1-m) y-u_{8}-w, a_{34}=-z, a_{41}=0, \quad a_{42}=0, a_{43}=u_{9} w, a_{44}=u_{9} z-u_{10} .
\end{aligned}
$$


The local stability analysis at $E_{0}$ :

The Jacobian matrix of system( 2 )at $E_{0}$ can be written as:

$$
J_{0}=J\left(E_{0}\right)=\left[\begin{array}{cccc}
-\left(u_{2}+u_{3}\right) & 1 & 0 & 0 \\
u_{2} & -u_{5} & 0 & 0 \\
0 & 0 & -u_{8} & 0 \\
0 & 0 & 0 & -a_{10}
\end{array}\right]
$$

Then the characteristic equation of $J\left(E_{0}\right)$ is given by:

$$
\left[\lambda^{2}+A \lambda+B\right]\left(-u_{8}-\lambda\right)\left(-u_{10}-\lambda\right)=0 \text {, }
$$

where:

$$
A=u_{2}+u_{3}+u_{5} \quad, \quad B=u_{5}\left(u_{2}+u_{3}\right)-u_{2},
$$

so, either

this gives two eigenvalues of $J_{0}$ by: $\lambda_{0 z}=-a_{3}<0$, and $\lambda_{0 w}=-a_{8}<0$.

Or

$$
\lambda^{2}+A \lambda+B=0
$$

where:

$\operatorname{tr}(A)=\lambda_{0 x}+\lambda_{0 y}=-\left(u_{2}+u_{3}+u_{5}\right)<0 \quad, \quad$ and $\quad \operatorname{det}(A)=\lambda_{0 x} \cdot \lambda_{0 y}=u_{5}\left(u_{2}+u_{3}\right)-$ $u_{2}$, which gives the other two eigenvalues of $J_{0}$ with negative real parts provided that the following condition holds:

$$
u_{5}>\frac{u_{2}}{\left(u_{2}+u_{3}\right)} \text {. }
$$

Then $E_{0}$ is locally asymptotically stable in the $R_{+}^{4}$. However, it is a saddle point otherwise.

\section{The local stability analysis at $E_{1}$}

The Jacobian matrix of system ( 2 ) at $E_{1}$ can be written as:

$$
J_{1}=J\left(E_{1}\right)==\left[b_{i j}\right]_{4 \times 4},
$$

where:

$b_{11}=-\left(u_{2}+u_{3}\right), \quad b_{12}=1-2 \bar{y}, b_{13}=-\frac{(1-m) \bar{x}}{u_{1}+\bar{x}}, b_{14}=0, b_{21}=u_{2}, \quad b_{22}=-u_{5}$,

$b_{23}-u_{4}(1-m) \bar{y}_{,} \quad b_{24}=0, \quad b_{31}=0, \quad b_{32}=0, \quad b_{33}=\frac{u_{6}(1-m) \bar{x}}{u_{1}+\bar{x}}+u_{7}(1-m) \bar{y}-u_{8}$,

$b_{34}=0, \quad b_{41}=0, b_{42}=0, \quad b_{43}=0, b_{44}=-u_{10}$.

Then the characteristic equation of $J_{1}$ is given by:

where:

$$
\left[\lambda^{2}+\bar{A} \lambda+\bar{B}\right]\left(b_{33}-\lambda\right)\left(-u_{10}-\lambda\right)=0
$$

$\bar{A}-\operatorname{tr}(A)=u_{2}+u_{3}+u_{5}$

$\bar{B}=\operatorname{det}(A)=u_{5}\left(u_{2}+u_{3}\right)-u_{2}+u_{2} \bar{y}$,

so, either

$$
\left(b_{33}-\lambda\right)\left(-u_{10}-\lambda\right)=0
$$

which gives two eigenvalues of $J_{1}$ by:

$\lambda_{1 z}=\frac{u_{6}(1-m) \bar{x}}{u_{1}+\bar{x}}+u_{7}(1-m) \bar{y}-u_{8}, \quad$ and $\lambda_{1 w}=-u_{10}<0$

Or $\quad \lambda^{2}+\bar{A} \lambda+\bar{B}=0$,

where: $\operatorname{tr}(A)=\lambda_{0 x}+\lambda_{0 y}=-\left(u_{2}+u_{3}+u_{5}\right)<0 \quad$, and $\operatorname{det}(A)=\lambda_{0 x} \cdot \lambda_{0 y}=u_{5}\left(u_{2}+u_{3}\right)-u_{2}+u_{2} \bar{y}$, which gives the other two eigenvalues of $J_{1}$ with negative real parts due to the condition( 3 ).

Then, $E_{1}$ is locally asymptotically stable in $R_{+}^{4}$ if in addition to the condition $(8 \mathrm{e})$, the following condition holds:

$$
u_{8}>\frac{u_{6}(1-m) \bar{x}}{u_{1}+\bar{x}}+u_{7}(1-m) \bar{y} .
$$

However, it is a saddle point otherwise.

The local stability analysis at $E_{2}$

The Jacobian matrix of system ( 2 ) at $E_{2}$ can be written as: 
where:

$$
J_{2}=J\left(E_{2}\right)=\left[c_{i j}\right]_{4 \times 4},
$$

$c_{11}=-\frac{u_{1}(1-m) \hat{z}}{\left(u_{1}+\hat{x}\right)^{2}}-\left(u_{2}+u_{3}\right)<0, \quad c_{12}=1-2 \hat{y}, \quad c_{13}=-\frac{(1-m) \hat{x}}{u_{1}+\hat{x}}<0, \quad c_{14}=0, \quad a_{21}=u_{2}>0$, $c_{22}=\frac{-u_{2} \hat{x}}{\hat{y}}<0, c_{23}-u_{4}(1-m) \hat{y}<0, \quad c_{24}=0, \quad c_{31}=\frac{u_{1} u_{6}(1-m) \hat{z}}{\left(u_{1}+\hat{x}\right)^{2}}>0, \quad c_{32}=u_{7}(1-m) \hat{z}>0$, $c_{33}=\frac{u_{6}(1-m) \hat{x}}{u_{1}+\hat{x}}+u_{7}(1-m) \hat{y}-u_{8}, \quad c_{34}=-\hat{z}<0, \quad c_{41}=0, \quad c_{42}=0, \quad c_{43}=0, \quad c_{44}=u_{9} \hat{z}-u_{10}$. Then the characteristic equation of $J_{2}$ is given by:

where:

$$
\left[\lambda^{3}+A_{1} \lambda^{2}+A_{2} \lambda+A_{3}\right]\left(u_{9} \hat{z}-u_{10}-\lambda\right)=0,
$$

$A_{1}=-\left(c_{11}+c_{22}\right)>0$

$A_{2}=c_{11} c_{22}-c_{12} c_{21}-c_{13} c_{31}-c_{23} c_{32}$

$A_{3}=c_{32}\left(c_{11} c_{23}-c_{13} c_{21}\right)+c_{22} c_{13} c_{31}-c_{12} c_{23} c_{31}$,

so, either

$$
u_{9} \hat{z}-u_{10}-\lambda=0
$$

$\operatorname{Or} \lambda^{3}+A_{1} \lambda^{2}+A_{2} \lambda+A_{3}=0$.

Hence from equation ( $9 c$ ) we obtain that:

$\lambda_{2 w}=u_{9} \hat{z}-u_{10}$, which is negative if the following condition holds:

$$
\hat{z}<\frac{u_{10}}{u_{9}}(9 e)
$$

Since $A_{1}>0$, then by using Routh-Hawirtiz criterion equation ( $9 d$ ) has roots (eigenvalues) with negative real parts if and only if

$A_{3}>0$ and $\Delta=A_{1} A_{2}-A_{3}>0$.

Straightforward computation shows that:

$$
\Delta=-\left(c_{11}+c_{22}\right) c_{11} c_{22}+c_{11}\left(c_{12} c_{21}+c_{13} c_{31}\right)+c_{22} c_{23} c_{32}+c_{12} c_{23} c_{31}+c_{21}\left(c_{22} c_{12}+c_{13} c_{32}\right) .
$$

Now, according to the form of $A_{3}$ and signs of the Jacobian elements all terms of $A_{3}$ will be positive under the following conditions:

$$
\begin{aligned}
& \hat{y}>\frac{1}{2} \\
& u_{7}>\frac{u_{1} u_{6}(2 \hat{y}-1)}{u_{1}(1-m) \hat{z}+\left(u_{2}+u_{3}\right)\left(u_{1}+\hat{x}\right)^{2}} .
\end{aligned}
$$

However $\Delta$ becomes positive, since the first four terms of $\Delta$ are positive, while the last one will be positive if and only if in addition to the condition ( $9 f$ ) the following condition holds:

$$
u_{7}<\frac{u_{2}(2 \hat{y}-1)\left(u_{1}+\hat{x}\right)}{(1-m)^{2} \hat{y} \hat{z}} \text {. }
$$

Therefore, all the eigenvalues of $J_{2}$ have negative real parts under the given conditions and hence $E_{2}$ is locally asymptotically stable. However, it is unstable otherwise.

The local stability analysis at $E_{3}$

The Jacobian matrix of system( 2 )at $E_{3}$ can be written as:

$$
J_{3}=\left[d_{i j}\right]_{4 \times 4},
$$

where:

$d_{11}=-\frac{u_{1}(1-m) z^{*}}{\left(u_{1}+x^{*}\right)^{2}}-\left(u_{2}+u_{3}\right)<0, \quad d_{12}=1-2 y^{*}, \quad d_{13}=-\frac{(1-m) x^{*}}{u_{1}+x^{*}}<0, d_{14}=0, d_{21}=u_{2}>0$,

$d_{22}=\frac{-u_{2} x^{*}}{\hat{y}}<0, \quad d_{23}-u_{4}(1-m) y^{*}<0, \quad d_{24}=0, \quad d_{31}=\frac{u_{1} u_{6}(1-m) z^{*}}{\left(u_{1}+x^{*}\right)^{2}}>0, \quad d_{32}=u_{7}(1-m) z^{*}>0$,

$d_{33}=\frac{u_{6}(1-m) x^{*}}{u_{1}+x^{*}}+u_{7}(1-m) y^{*}-u_{8}-w^{*}, d_{34}=-z^{*}<0, \quad d_{41}=0, \quad d_{42}=0, d_{43}=u_{9} w^{*}>0$ ，

$d_{44}=u_{9} z^{*}-u_{10}$.

Then the characteristic equation of $J_{3}$ is given by:

$$
\left[\lambda^{4}+B_{1} \lambda^{3}+B_{2} \lambda^{2}+B_{3} \lambda+B_{4}\right]=0,
$$


where:

$$
\begin{aligned}
& B_{1}=-\alpha_{0}>0 . \\
& B_{2}=\alpha_{1}-\alpha_{2}-\alpha_{3}-\alpha_{4}-\alpha_{5} . \\
& B_{3}=\left(\alpha_{0} \alpha_{5}-\alpha_{6}\right)+d_{11} \alpha_{4}+d_{22} \alpha_{3}-\alpha_{7} . \\
& B_{4}=\alpha_{5}\left(\alpha_{2}-\alpha_{1}\right)>0, \quad \text { with, } \\
& \alpha_{0}=\left(d_{11}+d_{22}\right)<0, \alpha_{1}=d_{11} d_{22}>0, \alpha_{2}=d_{12} d_{21}, \\
& \alpha_{3}=d_{13} d_{31}<0, \quad \alpha_{4}=d_{23} d_{32}<0, \alpha_{5}=d_{34} d_{43}<0, \\
& \alpha_{6}=d_{12} d_{23} d_{31}, \quad \alpha_{7}=d_{13} d_{21} d_{32}<0 .
\end{aligned}
$$

Now by using Routh-Hawirtiz criterion equation ( $10 b$ ) has roots (eigenvalues) with negative real parts if and only if $B_{i}>0, i=1,3,4$ and $\Delta=\left(B_{1} B_{2}-B_{3}\right) B_{3}-B_{1}^{2} B_{4}>0$.

Clearly $B_{1}>0$ and $B_{4}>0$ provided that:

$$
y^{*}>\frac{1}{2}
$$

Straightforward computation shows that:

$\Delta=\left[\propto_{0}\left(\propto_{2}-\propto_{1}\right)+\left(d_{11} \propto_{3}+\propto_{7}\right)+d_{22} \propto_{4}+\propto_{6}\right]\left[\left(\propto_{0} \propto_{5}-\propto_{6}\right)+\left(d_{11} \propto_{4}-\propto_{6}\right)+d_{22} \propto_{3}-\propto_{7}\right]$.

Now, according to the form of $B_{3}$ and signs of the Jacobian elements the last three terms of $B_{3}$ are positive, while the first one will be positive in addition to the condition $(10 c)$ the following condition holds:

$$
w^{*}>\frac{u_{1} u_{4} u_{6}(1-m)^{2}\left(2 y^{*}-1\right) y^{* 2}}{u_{9}\left[u_{1}(1-m) y^{*} z^{*}+\left[\left(u_{2}+u_{3}\right) y^{*}+u_{2} x^{*}\right]\left(u_{1}+x^{*}\right)^{2}\right]}
$$

On the other hand due to the conditions $(10 \mathrm{c})$ and $(10 \mathrm{~d})$, all elements of $\Delta$ are positive and the two elements $\left(d_{11} \propto_{3}+\propto_{7}\right),\left(d_{11} \propto_{4}-\propto_{6}\right)$ will be positive if the following condition holds:

$$
L_{1}<u_{7}<L_{2}
$$

where:

$$
L_{1}=\frac{u_{1} u_{6}\left(2 y^{*}-1\right)}{u_{1}(1-m) z^{*}+\left(u_{2}+u_{3}\right)\left(u_{1}+x^{*}\right)^{2}}, \quad \text { and } \quad L_{2}=\frac{u_{1} u_{6}\left[u_{1}(1-m) z^{*}+\left(u_{2}+u_{3}\right)\left(u_{1}+x^{*}\right)^{2}\right]}{u_{2}\left(u_{1}+x^{*}\right)^{4}} .
$$

Therefore, all the eigenvalues of $J_{3}$ have negative real parts under the given conditions and hence $E_{3}$ is locally asymptotically stable. However, it is unstable otherwise.

\section{The global stability analysis of system ( 2 )}

In this section the global stability analysis for the equilibrium points, which are locally asymptotically stable, of system( 2 )is studied analytically with the help of Lyapunov method as shown in the following theorems.

Theorem ( 2 ): Assume that the vanishing equilibrium point $E_{0}=(0,0,0,0)$ of system( 2 )is locally asymptotically stable in $R_{+}^{4}$. Then $E_{0}$ is globally asymptotically stable on the region $\omega_{0} \subset R_{+}^{4}$, where $\omega_{0}=$ $\left\{(x, y, z, w) \in R_{+}^{4}: y>1\right\}$.

Proof: Consider the following function:

$V_{0}(x, y, z, w)=x+y+z+w$,

Clearly $V_{0}: R_{+}^{4} \rightarrow R$ is a $C^{1}$ positive definite function.

Now by differentiating $V_{0}$ with respect to time $\mathrm{t}$ and doing some algebraic manipulation, gives that:

$$
\frac{d V_{0}}{d t}=y(1-y)-\left(1-u_{6}\right) \frac{(1-m) x z}{u_{1}+x}-\left(u_{4}-u_{7}\right) y z-\left(1-u_{9}\right) z w-u_{3} x-u_{5} y-u_{8} z-u_{10} w .
$$

Now, due to the facts that is mentioned in theorem (1), always $u_{6}<1, u_{9}<1$ and $u_{7}<u_{4}$, we get,

$\frac{d V_{0}}{d t}<y(1-y)-u_{3} x-u_{5} y-u_{8} z-u_{10} w$.

Hence $\frac{d V_{0}}{d t}<0$ in the region $\omega_{0}$ and then $V_{0}$ is strictly Lyapunov function. Thus we obtain that $E_{0}$ is a globally asymptotically stable in the region $\omega_{0}$, and the proof is complete.

Theorem (3): Assume that the free predators equilibrium point $E_{1}=(\bar{x}, \bar{y}, 0,0)$ of system( 2 )is a locally asymptotically stable in $R_{+}^{4}$. Then $E_{1}$ is a globally asymptotically stable on the region $\omega_{1} \subset R_{+}^{4}$ that satisfies the following conditions:

$$
\frac{1}{x}-\frac{(y+\bar{y})}{x}+\frac{u_{2} u_{7}}{u_{4} u_{6} y} \leq 2 \sqrt{\frac{u_{2} u_{7}\left(\bar{y}-\bar{y}^{2}\right)}{u_{4} u_{6} x y \bar{y}}}
$$


$\bar{x}<x$

Proof: Consider the following function

$V_{1}(x, y, z, w)=c_{1}\left(x-\bar{x}-\bar{x} \ln \frac{x}{\bar{x}}\right)+c_{2}\left(y-\bar{y}-\bar{y} \ln \frac{y}{\bar{y}}\right)+c_{3} z+c_{4} w$,

where: $c_{1}, c_{2}, c_{3}$ and $c_{4}$ are positive constants to be determined.

Clearly $V_{1}: R_{+}^{4} \rightarrow R$ is a $C^{1}$ positive definite function. Now by differentiating $V_{1}$ with respect to time $\mathrm{t}$ and doing some algebraic manipulation, gives that:

$$
\begin{aligned}
& \frac{d V_{1}}{d t}=-c_{1} \frac{\left(\bar{y}-\bar{y}^{2}\right)}{x \bar{x}}(x-\bar{x})^{2}-c_{2} \frac{u_{2} \bar{x}}{y \bar{y}}(y-\bar{y})^{2}+\left[c_{1}\left(\frac{1}{x}-\frac{(y+\bar{y})}{x}\right)+\frac{c_{2} u_{2}}{y}\right](x-\bar{x})(y-\bar{y}) \\
&-\left(c_{1}-c_{3} u_{6}\right) \frac{(1-m) x z}{u_{1}+x}-\left(c_{2} u_{4}-c_{3} u_{7}\right)(1-m) y z+\left[\frac{c_{1}(1-m) \bar{x}}{u_{1}+x}+c_{2} u_{4}(1-m) \bar{y}-c_{3} u_{8}\right] z \\
&-\left(c_{3}-c_{4} u_{9}\right) w z-c_{4} u_{10} w .
\end{aligned}
$$

By chosen $c_{1}=1, \quad c_{2}=\frac{u_{7}}{u_{4} u_{6}}, \quad c_{3}=\frac{1}{u_{6}}, \quad c_{4}=\frac{1}{u_{6} u_{9}}$ we get:

$$
\begin{aligned}
\frac{d V_{1}}{d t} \leq & -\frac{\left(\bar{y}-\bar{y}^{2}\right)}{x \bar{x}}(x-\bar{x})^{2}-\frac{u_{7} u_{2} \bar{x}}{u_{6} u_{4} y \bar{y}}(y-\bar{y})^{2}+\left[\left(\frac{1}{x}-\frac{(y+\bar{y})}{x}\right)+\frac{u_{7} u_{2}}{u_{6} u_{4} y}\right](x-\bar{x})(y-\bar{y}) \\
& -\left[\frac{u_{8}}{u_{6}}-\frac{(1-m) \bar{x}}{u_{1}+x}+\frac{u_{7}(1-m) \bar{y}}{u_{6}}\right] z-\frac{u_{10}}{u_{6} u_{9}} w .
\end{aligned}
$$

Now by using the conditions $(11 a),(11 b)$ and $(11 c)$ we obtain that:

$\frac{d V_{1}}{d t}<-\left[\sqrt{\frac{\left(\bar{y}-\bar{y}^{2}\right)}{x \bar{x}}}(x-\bar{x})-\sqrt{\frac{u_{2} u_{7} \bar{x}}{u_{6} u_{4} y \bar{y}}}(y-\bar{y})\right]-\left[\frac{u_{8}}{u_{6}}-\frac{(1-m) \bar{x}}{u_{1}+\bar{x}}+\frac{u_{7}(1-m) \bar{y}}{u_{6}}\right] z-\frac{u_{10}}{u_{6} u_{9}} w^{2}{ }^{2}$

Clearly, $\frac{d V_{1}}{d t}$ is negative definite on the region $\omega_{1}$ due to the local stability condition $(8 e)$. Hence $V_{1}$ is strictly Lyapunov function. thus $E_{1}$ is a globally asymptotically stable on the region $\omega_{1}$ and the proof is complete .

Theorem (4): Assume that the free top predators' equilibrium point $E_{2}=(\hat{x}, \hat{y}, \hat{z}, 0)$ of system ( 2 ) is locally asymptotically stable in $R_{+}^{4}$. Then $E_{2}$ is a globally asymptotically stable on any region $\omega_{2} \subset R_{+}^{4}$ that satisfies the following conditions:

$$
\mu_{1} \leq 2 \sqrt{\frac{u_{1} u_{2} u_{6} u_{7} \hat{x}}{u_{4}\left(u_{1}+\hat{x}\right) y \hat{y}}\left(\mu_{2}-\mu_{3}\right)}
$$

$$
\mu_{3}<\mu_{2}
$$

where:

$$
\mu_{1}=\frac{u_{1} u_{6}}{u_{1}+\hat{x}}\left(\frac{1}{x}-\frac{(y+\hat{y})}{x}\right)+\frac{u_{2} u_{7}}{u_{4} y}, \quad \mu_{2}=\frac{\left(\hat{y}-\hat{y}^{2}\right)}{x \hat{x}}, \quad \mu_{3}=\frac{(1-m) \hat{z}}{\left(u_{1}+\hat{x}\right)\left(u_{1}+x\right)} .
$$

Proof: Consider the following function:

$$
V_{2}(x, y, z, w)=c_{1}\left(x-\hat{x}-\hat{x} \ln \frac{x}{\hat{x}}\right)+c_{2}\left(y-\hat{y}-\hat{y} \ln \frac{y}{\hat{y}}\right)+c_{3}\left(z-\hat{z}-\hat{z} \ln \frac{z}{\hat{z}}\right)+c_{4} w,
$$

where: $c_{1}, c_{2}, c_{3}$ and $c_{4}$ are positive constants to be determined.

Clearly $V_{2}: R_{+}^{4} \rightarrow R$ is a $C^{1}$ positive definite function. Now by differentiating $V_{2}$ with respect to time $\mathrm{t}$ and doing some algebraic manipulation, gives that:

$$
\begin{aligned}
\frac{d V_{2}}{d t}=- & c_{1}\left(\frac{\left(\hat{y}-\hat{y}^{2}\right)}{x \hat{x}}-\frac{(1-m) \hat{z}}{\left(u_{1}+\hat{x}\right)\left(u_{1}+x\right)}\right)(x-\hat{x})^{2}-\left(c_{1}-\frac{c_{3} u_{1} u_{6}}{u_{1}+\hat{x}}\right) \frac{(1-m)}{\left(u_{1}+x\right)}(x-\hat{x})(z-\hat{z}) \\
+ & {\left[c_{1}\left(\frac{1}{x}-\frac{(y+\hat{y})}{x}\right)+\frac{c_{2} u_{2}}{y}\right](x-\hat{x})(y-\hat{y})-c_{2} \frac{u_{2} \hat{x}}{y \hat{y}}(y-\hat{y})^{2} } \\
& -\left(c_{2} u_{4}-c_{3} u_{7}\right)(1-m)(y-\hat{y})\left(z-\hat{z}-\left(c_{3}-c_{4} u_{9}\right) w z-\left(c_{4} u_{10}-c_{3} \hat{z}\right) w .\right.
\end{aligned}
$$

By chosen $\quad c_{1}=\frac{u_{1} u_{6}}{u_{1}+\hat{x}}, \quad c_{2}=\frac{u_{7}}{u_{4}}, \quad c_{3}=1, \quad c_{4}=\frac{1}{u_{9}}$ we get: 


$$
\begin{aligned}
\frac{d V_{2}}{d t} \leq- & \frac{u_{1} u_{6}}{u_{1}+\hat{x}}\left(\frac{\left(\hat{y}-\hat{y}^{2}\right)}{x \hat{x}}-\frac{(1-m) \hat{z}}{\left(u_{1}+\hat{x}\right)\left(u_{1}+x\right)}\right)(x-\hat{x})^{2}-\frac{u_{2} u_{7} \hat{x}}{u_{4} y \hat{y}}(y-\hat{y})^{2} \\
& +\left[\frac{u_{1} u_{6}}{u_{1}+\hat{x}}\left(\frac{1}{x}-\frac{(y+\hat{y})}{x}\right)+\frac{u_{2} u_{7}}{u_{4} y}\right](x-\hat{x})(y-\hat{y})-\left(u_{10}-u_{9} \hat{z}\right) w .
\end{aligned}
$$

Now by using the conditions $(12 a)$ and $(12 b)$ we obtain that:

$\frac{d V_{2}}{d t}<-\frac{u_{1} u_{6}}{u_{1}+\hat{x}}\left[\sqrt{\frac{\left(\hat{y}-\hat{y}^{2}\right)}{x \hat{x}}-\frac{(1-m) \hat{z}}{\left(u_{1}+\hat{x}\right)\left(u_{1}+x\right)}}(x-\hat{x})-\sqrt{\frac{u_{2} u_{7} \hat{x}}{u_{4} y \hat{y}}}(y-\hat{y})\right]^{2}-\left(u_{10}-u_{9} \hat{z}\right) w$.

Clearly, $\frac{d V_{2}}{d t}$ is negative definite on the region $\omega_{2}$ due to the conditions $(12 c)$ and the local stability condition $(9 e)$. Hence $V_{2}$ is strictly Lyapunov function. thus $E_{2}$ is a globally asymptotically stable on the region $\omega_{2}$ and the proof is complete.

Theorem (5): Assume that the positive (coexistence) equilibrium point $E_{3}\left(x^{*}, y^{*}, z^{*}, w^{*}\right)$ of system ( 2 ) is locally asymptotically stable in the $R_{+}^{4}$. Then $E_{3}$ is a globally asymptotically stable on any region $\omega_{3} \subset R_{+}^{4}$ that satisfies the following conditions:

$$
\frac{u_{1} u_{6}}{u_{1}+x^{*}}\left(\frac{1}{x}-\frac{\left(y+y^{*}\right)}{x}\right)+\frac{u_{2} u_{7}}{u_{4} y} \leq 2 \sqrt{\frac{u_{1} u_{2} u_{6} u_{7} x^{*}\left(\beta_{1}-\beta_{2}\right)}{u_{4}\left(u_{1}+x^{*}\right) y y^{*}}}
$$

$$
z<\frac{u_{10}}{u_{9}}
$$

$$
w^{*}<w
$$

$$
\beta_{2}<\beta_{1}, \quad(13 e)
$$

where:

$\beta_{1}=\frac{\left(y^{*}-y^{* 2}\right)}{x x^{*}}, \quad \beta_{1}=\frac{(1-m) z^{*}}{\left(u_{1}+x^{*}\right)\left(u_{1}+x\right)}$.

Proof: Consider the following function:

$$
\begin{aligned}
V_{3}(x, y, z, w)= & c_{1}\left(x-x^{*}-x^{*} \ln \frac{x}{x^{*}}\right)+c_{2}\left(y-y^{*}-y^{*} \ln \frac{y}{y^{*}}\right)+c_{3}\left(z-z^{*}-z^{*} \ln \frac{z}{z^{*}}\right) \\
& +c_{4}\left(w-w^{*}-w^{*} \ln \frac{w}{w^{*}}\right),
\end{aligned}
$$

where: $c_{1}, c_{2}, c_{3}$ and $c_{4}$ are positive constants to be determined.

Clearly $V_{3}: R_{+}^{4} \rightarrow R$ is a $C^{1}$ positive definite function. Now differentiating by $V_{3}$ with respect to time $\mathrm{t}$ and doing some algebraic manipulation, gives that:

$$
\begin{aligned}
\frac{d V_{3}}{d t}=-c_{1} & \left(\frac{\left(y^{*}-y^{* 2}\right)}{x x^{*}}-\frac{(1-m) z^{*}}{\left(u_{1}+x^{*}\right)\left(u_{1}+x\right)}\right)\left(x-x^{*}\right)^{2}-\frac{c_{2} u_{2} x^{*}}{y y^{*}}\left(y-y^{*}\right)^{2} \\
+ & {\left[c_{1}\left(\frac{1}{x}-\frac{\left(y+y^{*}\right)}{x}\right)+\frac{c_{2} u_{2}}{y}\right]\left(x-x^{*}\right)\left(y-y^{*}\right)-\left(c_{1}-\frac{c_{3} u_{1} u_{6}}{u_{1}+x^{*}}\right) \frac{(1-m)}{\left(u_{1}+x\right)}\left(x-x^{*}\right)\left(z-z^{*}\right) } \\
& -\left(c_{2} u_{4}-c_{3} u_{7}\right)(1-m)\left(y-y^{*}\right)\left(z-z^{*}\right)-\left(c_{3}-c_{4} u_{9}\right)\left(z-z^{*}\right)\left(w-w^{*}\right) .
\end{aligned}
$$

By choosing $c_{1}=\frac{u_{1} u_{6}}{u_{1}+x^{*}}, \quad c_{2}=\frac{u_{7}}{u_{4}}, \quad c_{3}=c_{4}=1$, we get:

$$
\begin{aligned}
\frac{d V_{3}}{d t} \leq- & \frac{u_{1} u_{6}}{u_{1}+x^{*}}\left(\frac{\left(y^{*}-y^{* 2}\right)}{x x^{*}}-\frac{(1-m) z^{*}}{\left(u_{1}+x^{*}\right)\left(u_{1}+x\right)}\right)\left(x-x^{*}\right)^{2} \\
& +\left[\frac{u_{1} u_{6}}{u_{1}+x^{*}}\left(\frac{1}{x}-\frac{\left(y+y^{*}\right)}{x}\right)+\frac{u_{2} u_{7}}{u_{4} y}\right]\left(x-x^{*}\right)\left(y-y^{*}\right) \\
& -\frac{u_{2} u_{7} x^{*}}{u_{4} y y^{*}}\left(y-y^{*}\right)^{2}-\left(1-u_{9}\right)\left(z-z^{*}\right)\left(w-w^{*}\right)
\end{aligned}
$$

Now by using the conditions( $13 a$ ) and $(13 b)$ we obtain that: 
$\frac{d V_{3}}{d t}<-\left[\sqrt{\frac{u_{1} u_{6}}{u_{1}+x^{*}}\left(\beta_{1}-\beta_{2}\right)}\left(x-x^{*}\right)-\sqrt{\frac{u_{2} u_{7} x^{*}}{u_{4} y y^{*}}}\left(y-y^{*}\right)\right]^{2}-\left(1-u_{9}\right)\left(z-z^{*}\right)\left(w-w^{*}\right)$.

Clearly, $\frac{d V_{3}}{d t}$ is negative definite ontheregion $\omega_{3}$ due to the conditions $(13 c),(13 d),(13 e)$ and the fact that is mentioned intheorem $(1), u_{9}<1$.

Hence $V_{3}$ is strictly Lyapunov function. Thus $E_{3}$ is a globally asymptotically stable on the region $\omega_{3}$ and the proof is complete .

\section{Numerical analysis of system(2)}

In this section, the dynamical behavior of system ( 2 ) is studied numerically for different sets of parameters and different sets of initial points. The objectives of this study are: first investigate the effect of varying the value of each parameter on the dynamical behavior of system (2) and second confirm our obtained analytical results. It is observed that, for the following set of hypothetical parameters that satisfies stability conditions of the positive equilibrium point, system (2) has a globally asymptotically stable positive equilibrium point as shown in Fig. (6.1).

$$
\begin{aligned}
& u_{1}=0.6, u_{2}=0.4, u_{3}=0.1, u_{4}=0.5, u_{5}=0.1, u_{6}=0.3 \\
& u_{7}=0.3, u_{8}=0.1, u_{9}=0.5, u_{10}=0.1, m=0.5 .
\end{aligned}
$$
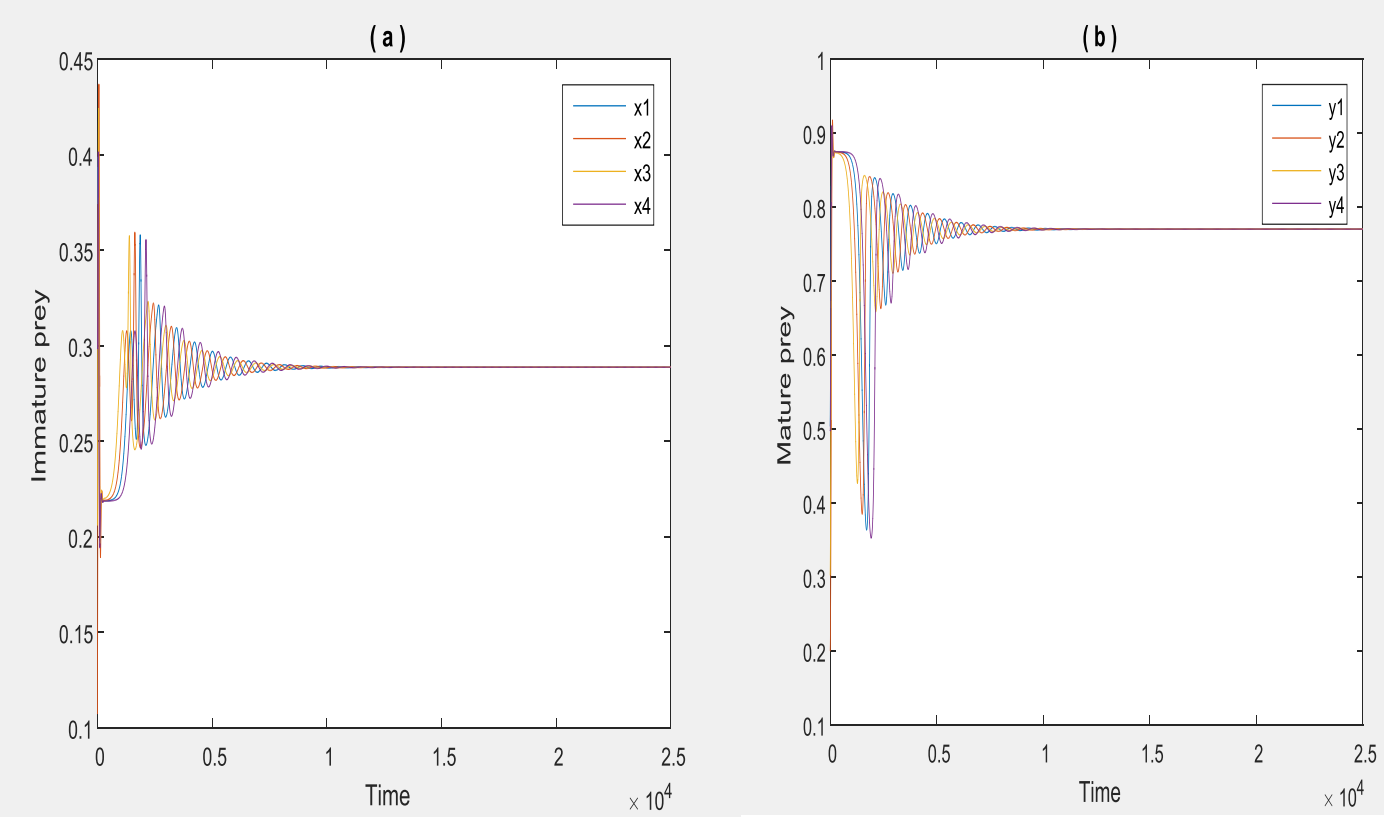

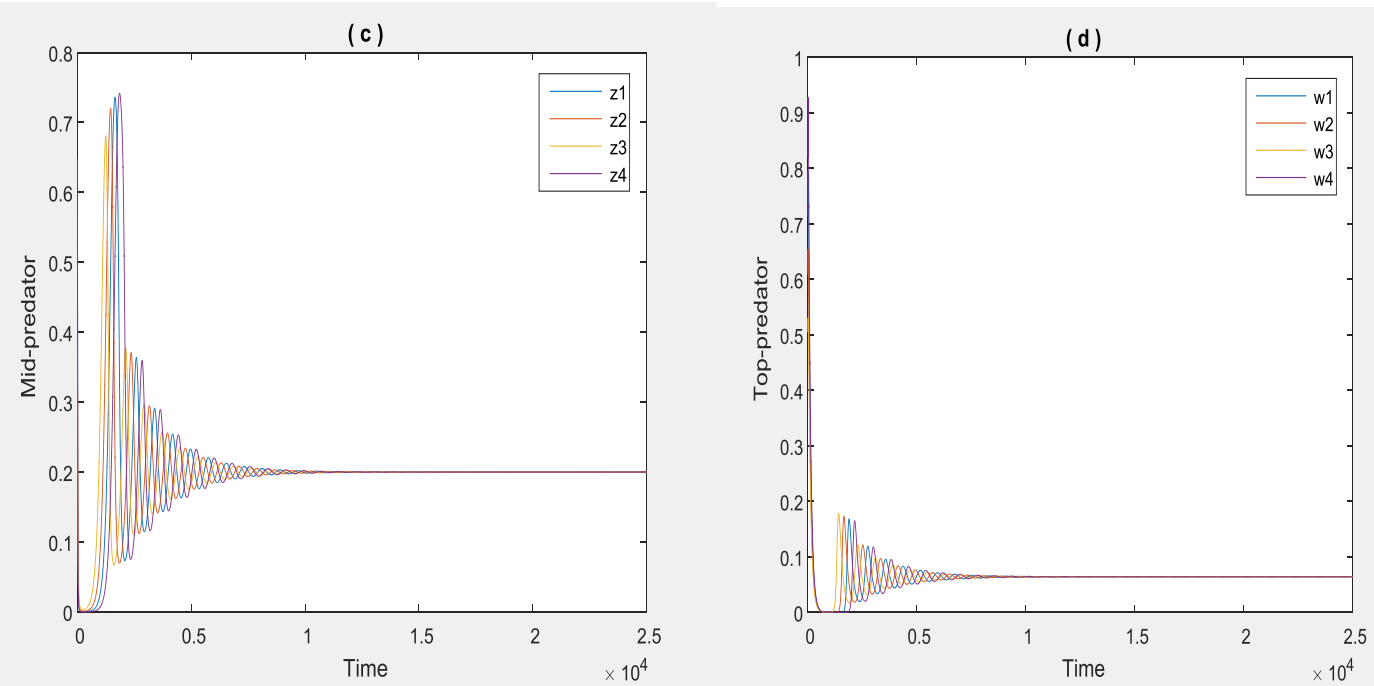

Fig.(6.1):-The time series of the solution of system(2)started from the four different initial points $(0.4,0.5,0.6,0.7),(0.1,0.2,0.5,0.6),(0.2,0.3,0.4,0.5)$, and $(0.3,0.5,0.7,0.8)$, for the data given by(6.1) (a), the trajectories of $x$ as a function of time, (b) the trajectories of $y$ as a function of time, (c) trajectories of $\mathrm{z}$ as a function of time, (d) the trajectories of $\mathrm{w}$ as a function of time.

Clearly, Fig. (6.1) shows that system(2) has a globally asymptotically stable as the solution of system( 2 )approaches asymptotically to the positive equilibrium point $E_{3}=(0.28,0.77,0.2,0.06)$ starting from four different initial points and this is confirming our obtained analytical results.

Now, in order to discuss the effect of the parameters values of system( 2 )on the dynamical behavior of the system, the system is solved numerically for the data given in( 6.1 )with varying one parameter at each time.

By varying the parameter $u_{1}$ which represents the half saturation rate of the mid-predator upon the immature prey and keeping the rest of parameters as data given in(6.1) in the range $0.01 \leq u_{1}<2$, it is observed that the solution of system( 2 )approaches asymptotically to the positive equilibrium point $E_{3}$. , as shown in Fig.( 6.2 ), for typical value $u_{1}=0.4$.

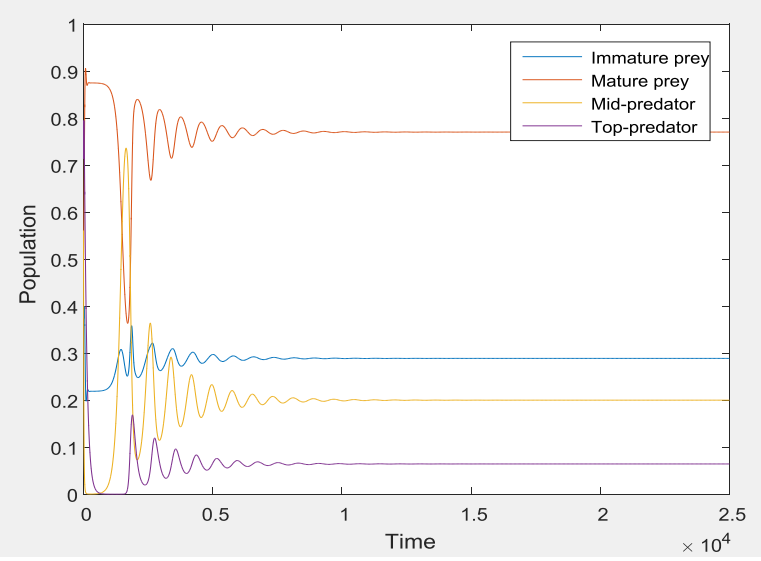

Fig. ( 6.2 ):-Time series of the solution of system (2) for the data given in(6.1) with $\mathrm{u}_{1}=0.4$, which approaches to $E_{3}=(0.28,0.75,0.2,0.07)$ in the interior of $\mathrm{R}_{+}^{4}$.

Now, varying the growth rate parameter of immature prey $u_{2}$ and keeping the rest of parameters values as data given in (6.1), it is observed that for $0.1 \leq u_{2}<2$ the solution of system ( 2 ) approaches asymptotically to a positive equilibrium point $E_{3}$. 
On the other hand varying the natural death rate of immature prey parameter $u_{3}$ and keeping the rest of parameters values as data in (6.1), it is observed that for $0.01 \leq u_{3}<0.90$ the solution of system (2)approaches asymptotically to the positive equilibrium point $E_{3}$, as shown in Fig. ( 6.3 ) $a$, for typical value $u_{3}=0.6$, while increasing this parameter for $0.90 \leq u_{3}<1$ causes extinction in the top-predator and the solution of system (2) approaches asymptotically to $E_{2}=(\hat{x}, \hat{y}, \hat{z}, 0)$ in the interior of the positive quadrant ofxyz - space, as shown in Fig. ( 6.3 ) $b$, for typical value $u_{3}=0.95$.

(a)

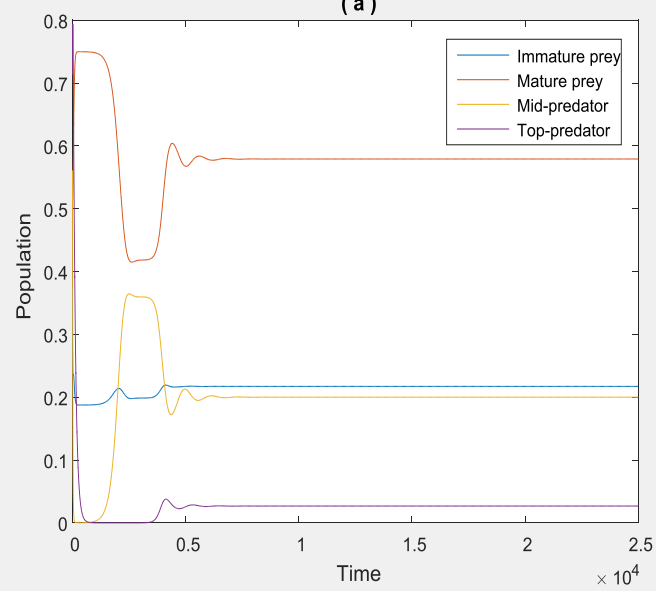

(b)

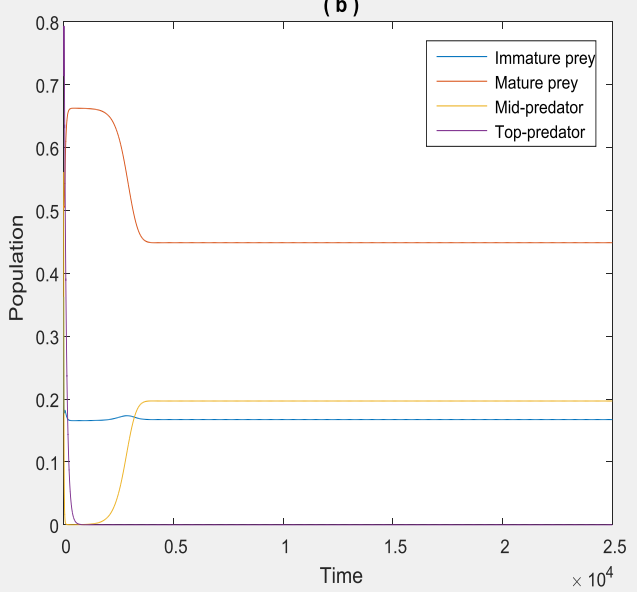

Fig (6.3) (a) :-Time series of the solution of system (2) for the data given by $(6.1)$ with $\mathrm{u}_{3}=0.6$, which approaches to $E_{3}=(0.21,0.57,0.2,0.02)$ in the interior of $\mathrm{R}_{+}^{4}$, and $(b)$ : Time series of the solution of system (2) for the data given by $(6.1)$ with $u_{3}=0.95$, which approaches to $E_{2}=(0.16,0.44,0.19,0)$ in the interior of the positive quadrant of $x y z-$ space .

Moreover, varying the parameter $\mathrm{r}_{4}$ which represents the predation rate of the mid-predator upon the immature prey, and keeping the rest of parameters values as data given in( 6.1), it is observed that for $0.01 \leq u_{4}<1.5$ the solution of system( 2 )still approaches asymptotically to a positive equilibrium point $E_{3}$.

The effect of varying the mature prey natural death rate parameter $u_{5}$ with $0.01 \leq u_{5}<0.41$ and keeping the rest parameters values as data given in (6.1), it observed that the solution of system ( 2 ) approaches asymptotically to a positive equilibrium point $E_{3}$, for typical value $u_{5}=0.3$ as shown in Fig. (6.4) $a$, however increasing this parameter in the range $0.41 \leq u_{5}<0.58$ causes extinction in the top-predatorand the solution of system (2) approaches asymptotically $\operatorname{toE}_{2}=(\hat{x}, \hat{y}, \hat{z}, 0)$ in the interior of the positive quadrant of xyz - space, as shown in Fig. (6.4) $b$, for typical value $u_{5}=0.5$, further increasing in the range $0.58 \leq$ $u_{5}<0.8$ causes extinction in the mid-predator and the solution of system ( 2 ) approaches asymptotically to the free predators equilibrium point $E_{1}=(\bar{x}, \bar{y}, 0,0)$ in the interior of the positive quadrant of $x y-$ plane, as shown in Fig. (6.4) $c$, for typical value $u_{5}=0.77$, then more increasing of this parameter in the range $0.8 \leq u_{5}<1$ causes extinction in all species and the solution of system (2) approaches asymptotically to the vanishing equilibrium point $\mathrm{E}_{0}=(0,0,0)$, as shown in $\operatorname{Fig}(6.4) d$, for typical value $u_{5}=0.9$.
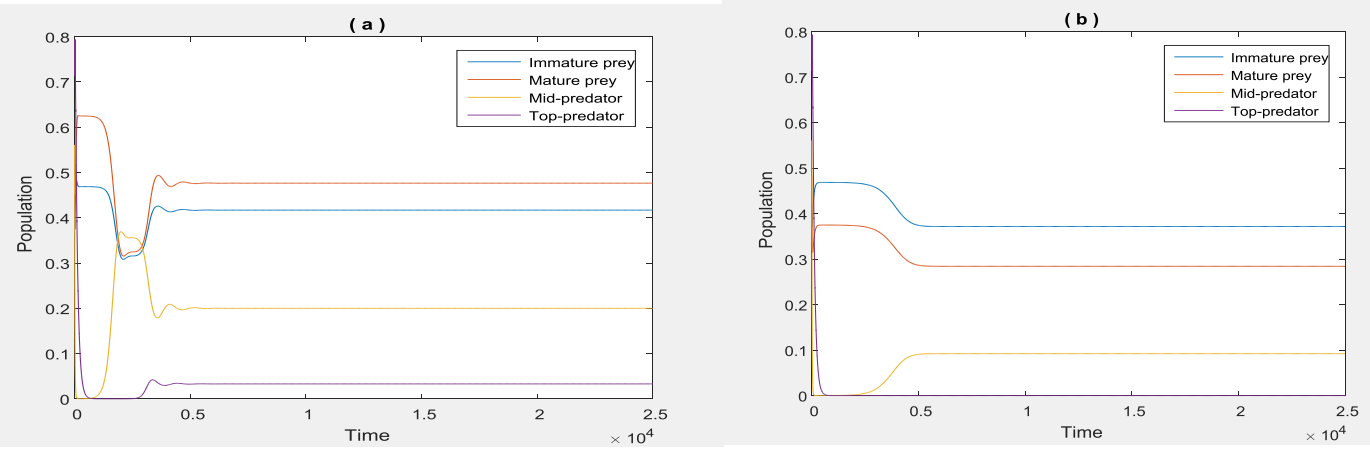

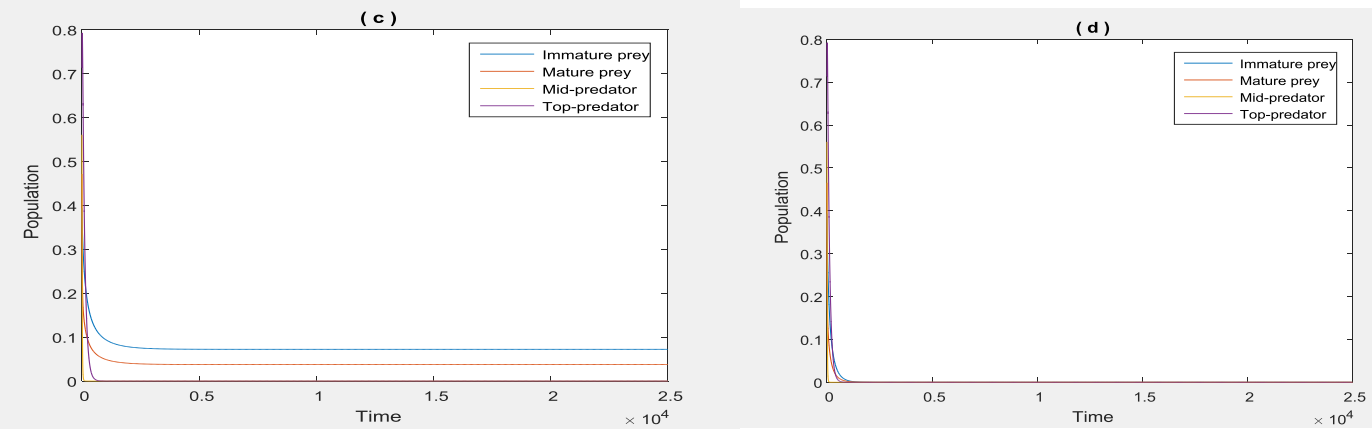

$\operatorname{Fig}(6.4)(\mathbf{a})$ :- Time series of the solution of system (2) for the data given by(6.1) with $\mathrm{u}_{5}=0.3$, which approaches toE $\mathrm{E}_{3}=(0.41,0.47,0.2,0.03)$ in the interior of $\mathrm{R}_{+}^{4},(\mathrm{~b})$ : Time series of the solution of system (2) for the data given by $(6.1)$ with $\mathrm{u}_{5}=0.5$, which approaches $\operatorname{toE}_{2}=(0.37,0.28,0.09,0)$ in the positive quadrant of xyz - space, ( c ): Time series of the solution of system (2) for the data given by(6.1)with $u_{5}=0.77$, which approaches to $E_{1}=(0.07,0.03,0,0)$ in the positive quadrant ofxy - plane, $(d)$ : Time series of the solution of system (2) for the data given by $(6.1)$ with $\mathrm{u}_{5}=0.9$, which approaches to $\mathrm{E}_{0}=(0,0,0,0)$.

The varying of the parameter $u_{6}$ which represents the conversion rate from the mature prey to the mid-predator, and keeping the rest of parameters values as data given in( 6.1), it is observed that for $0.01 \leq u_{6}<1$ the solution of system( 2 ) still approaches asymptotically to a positive equilibrium point $E_{3}$.

For varying the conversion rate parameter from the mature prey to the mid-predator $u_{7}$, with $0.01 \leq u_{7}<0.15$ the solution of system ( 2 ) approaches asymptotically to the positive free predators equilibrium point $\mathrm{E}_{1}=(\bar{x}, \bar{y}, 0,0)$ in the interior of the positive quadrant of $x y$ - plane, as shown in Fig.( 6.5) $a$, for typical value $u_{7}=0.1$, while for $u_{7}=0.15$ the solution of system (2) approaches asymptotically toE $E_{2}=(\hat{x}, \hat{y}, \hat{z}, 0)$ in the interior of the positive quadrant of xyz - space, as shown in Fig. (6.5) $b$, which means revival of the mid-predator population, then increasing this parameter in the range $0.15 \leq u_{7}<0.21$ leads revival of the top-predator and a small periodic attractor appears, as shown in Fig.(6.5) $c$, for typical value $u_{7}=0.16$ for more increasing in the range $0.21 \leq u_{7}<0.5$ the solution of system ( 2 ) approaches asymptotically to a positive equilibrium point $E_{3}$, for typical value $u_{7}=0.22$, as shown in Fig. (6.5) $d$.
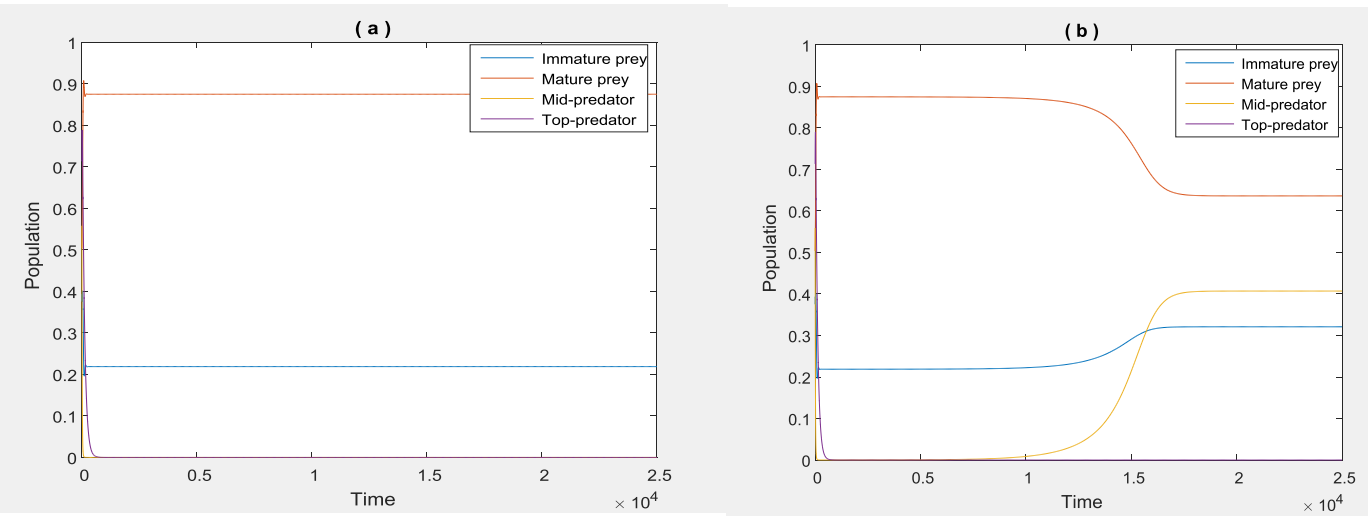

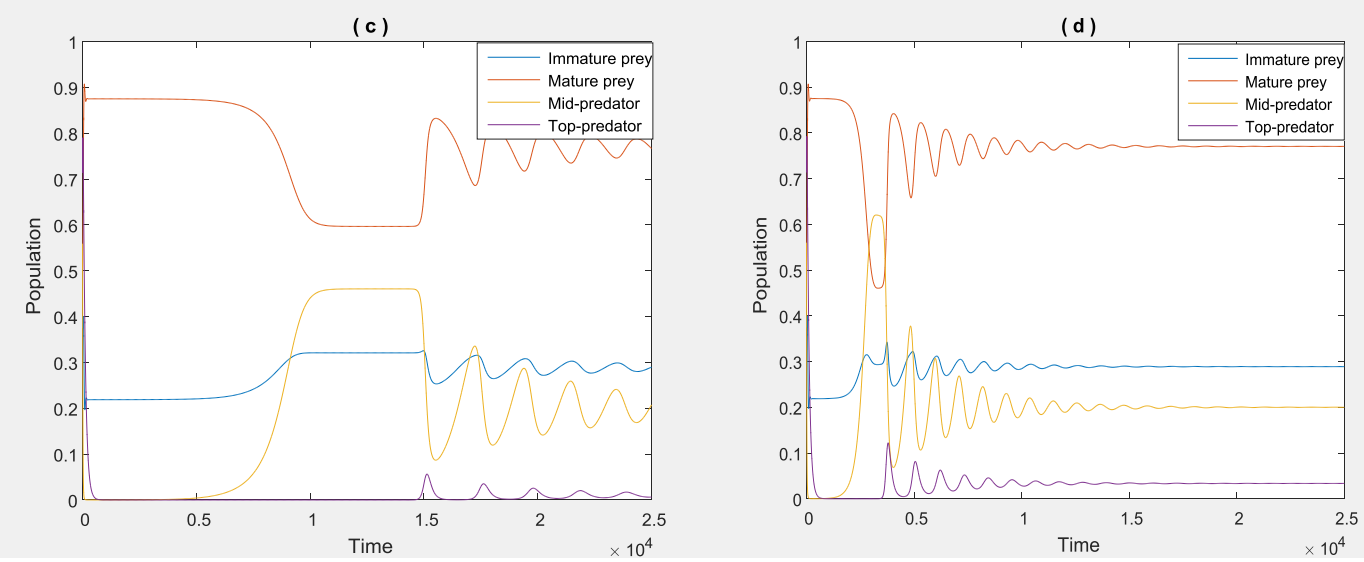

Fig (6.5 ):- ( a )Time series of the solution of system (2) for the data given by(6.1) with different values of $\mathrm{u}_{7},($ a $): E_{1}=(0.21,0.87,0,0)$ is a asymptotically stable with $u_{7}=0.1,(\mathrm{~b}): \mathrm{E}_{2}=(0.32,0.63,0.4,0)$ is a asymptotically stable with $\mathrm{u}_{7}=0.15,(\mathrm{c})$ : periodic attractor withu $\mathrm{u}_{7}=0.16,(\mathrm{~d})$ : $\mathrm{E}_{3}=$ $(0.28,0.77,0.19,0.03)$ is a asymptotically stable with $u_{7}=0.22$.

The varying of the mid-predator natural death rate parameter $u_{8}$, it is observed that for $0.01 \leq u_{8}<0.16$ the solution of system( 2 ) approaches asymptotically to the positive equilibrium point $E_{3}$, further increasing of this parameter with $u_{8}=0.16$ which causes extinction in the top-predator and the solution of system (2) approaches asymptotically to $E_{2}=(\hat{x}, \hat{y}, \hat{z}, 0)$ in the interior of the positive quadrant of $x y z-$ space, as shown in Fig.( 6.6 ) $a$, for typical value $u_{8}=0.16$. While for $0.16<u_{8}<1$ causes the extinction of the mid -predator the solution of system ( 2 ) approaches asymptotically $\mathrm{E}_{1}=(\bar{x}, \bar{y}, 0,0)$ in the interior of the positive quadrant of xy - plane,as shown in Fig. ( 6.6) $b$, for typical value $u_{8}=0.25$.
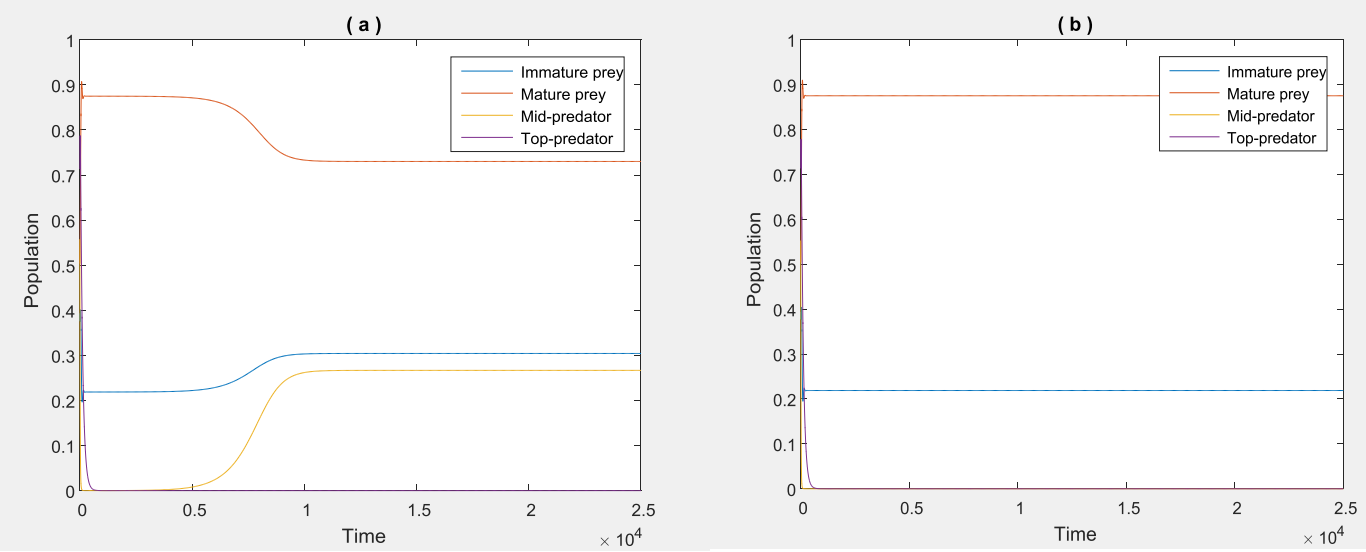

Fig( 6.6) (a ):- Time series of the solution of system (2) for the data given by(6.1) with $\mathrm{u}_{8}=0.16$, which approaches to $E_{2}=(0.30,0.73,0.26,0)$ in the interior of the positive quadrant of xyz - space, ( b ) : Time series of the solution of system (2) for the data given by(6.1) with $\mathrm{u}_{8}=0.25$, which approaches to $\mathrm{E}_{1}=(0.21,0.87,0,0)$ in the interior of the positive quadrant of $\mathrm{xy}-$ plane .

On the other hand, the varying of predation rate parameter of the top-predator upon the mid-predator, for0.01 $\leq$ $u_{9}<0.15$ the solution of system (2) approaches asymptotically toE $\mathrm{E}_{2}=(\hat{x}, \hat{y}, \hat{z}, 0)$ in the interior of the positive quadrant of xyz - space, as shown in Fig. (6.7) $a$, for typical value $u_{9}=0.1$, while for $0.15 \leq u_{9}<1$ the toppredator population revives and the solution of system (2) approaches asymptotically to a positive equilibrium point $E_{3}$, as shown in Fig. (6.7) $b$, for typical value $u_{9}=0.5$. 

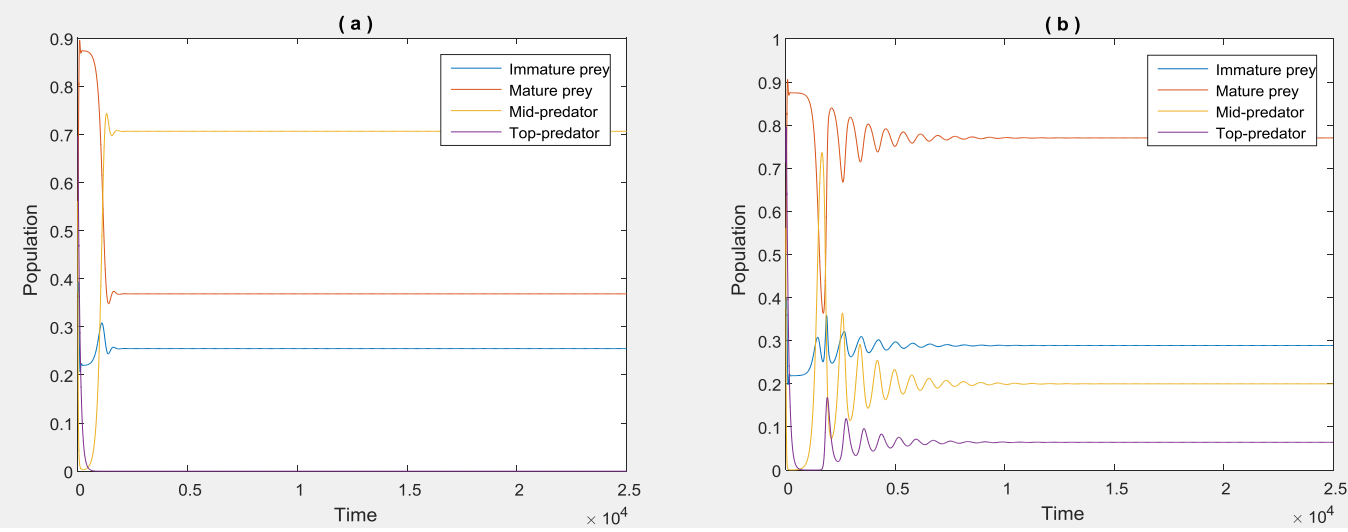

Fig( 6. 7 ):- ( a ) Time series of the solution of system (2) for the data given by(6.1) with $u_{9}=0.1$, which approaches to $E_{2}=(0.25,0.36,0.7,0)$ in the interior of the positive quadrant ofxyz - space, ( $b$ ):Time series of the solution of system (2) for thedatagivenin $(6.1)$ withu $_{9}=0.5$, whichapproachesto $E_{3}=(0.28,0.77,0.2,0.06)$ in the interior of $\mathrm{R}_{+}^{4}$.

Moreover, increasing the natural death rate of top-predator parameter $u_{10}$ in the range $0.1 \leq u_{10}<0.35$, the solution of system ( 2 ) approaches asymptotically to a positive equilibrium point $E_{3}$, as shown in Fig. (6.8) $a$, for typical value $u_{10}=0.3$, while the increasing of this parameter for $0.35 \leq u_{10}<1$ causes extinction of the toppredator population and the solution of system (2) approaches asymptotically toE $E_{2}=(\hat{x}, \hat{y}, \hat{z}, 0)$ in the interior of the positive quadrant of xyz - space, as shown in Fig.(6.8) $b$, for typical value $u_{10}=0.5$.

(a)

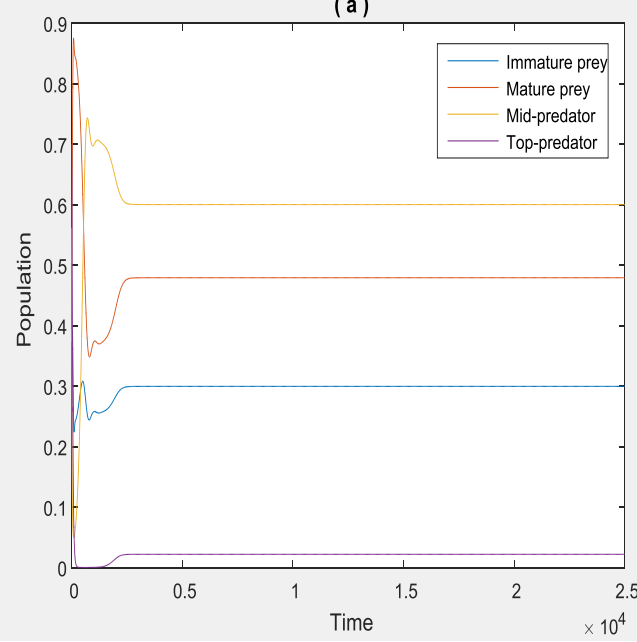

(b)

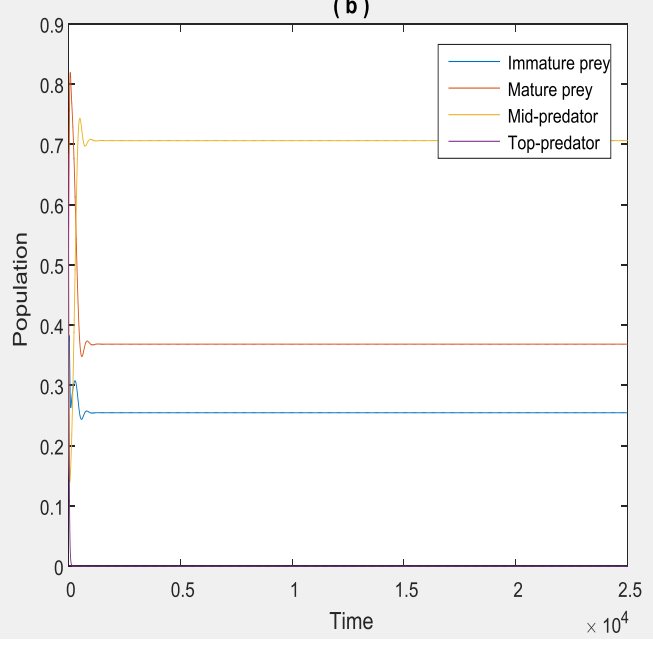

Fig.( 6.8 ):-( a ): Time series of the solution of system (2) for the data givenby(6.1)with $\mathrm{u}_{10}=0.3$, which approaches to $E_{3}=(0.29,0.47,0.6,0.02)$ in the interior of $R_{+}^{4}$. ( b ): Time series of the solution of system (2) for the data given by $(6.1)$ with $\mathrm{u}_{10}=0.5$, which approaches to $\mathrm{E}_{2}=(0.25,0.36,0.7,0)$ in the interior of the positive quadrant of $x y z-$ space.

Finally, varying the number of prey inside the refuge parameter $m$ and keeping the rest of parameters values as data given in ( 6.1 ), it is observed that for $0.01 \leq m<0.63$ the solution of system( 2 )approaches asymptotically to the positive equilibrium point $E_{3}$, as shown in Fig. (6.9) $a$, for typical value $m=0.5$, while increasing this parameter in the range $0.63 \leq m<0.69$ leads that the solution of system ( 2 ) approaches asymptotically to a periodic dynamics in Int. $R_{+}^{4}$, as shown in Fig. (6.9) $b$, for typical value $m=0.67$, more increasing of this parameter in the range $0.69 \leq \mathrm{m}<0.71$ causes extinction of the top-predator population and the solution of system (2) approaches asymptotically to the free top-predator equilibrium point $\mathrm{E}_{2}=(\hat{x}, \hat{y}, \hat{z}, 0)$ in the interior of the positive quadrant of xyz - space.as shown in Fig. (6.9) $c$, for typical value $m=0.7$ and for $0.71 \leq m<1$ the solution of system (2) 
approaches asymptotically to the free predators' equilibrium point $\mathrm{E}_{1}=(\bar{x}, \bar{y}, 0,0)$ in the interior of the positive quadrant of $\mathrm{xy}$ - plane, as shown in Fig.(6.9)d, for typical value $m=0.9$.

(a)

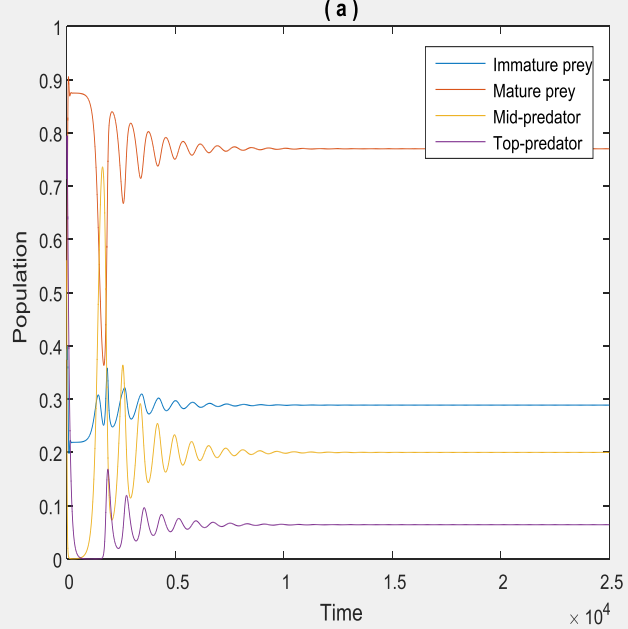

(c)

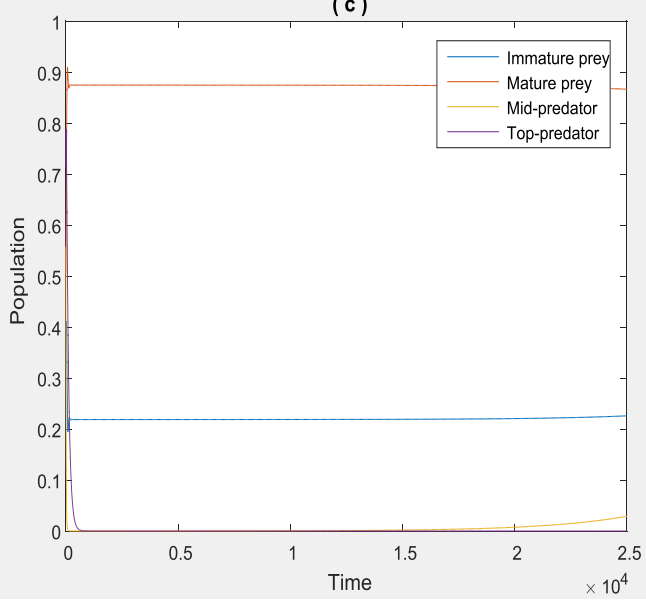

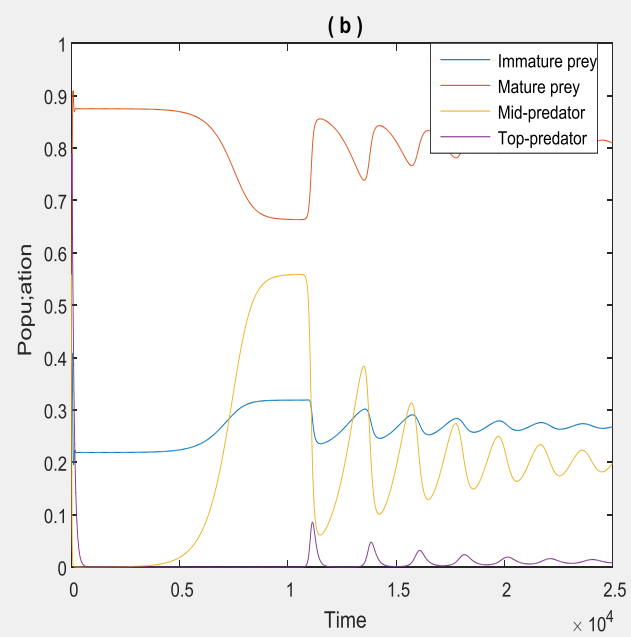

(d)

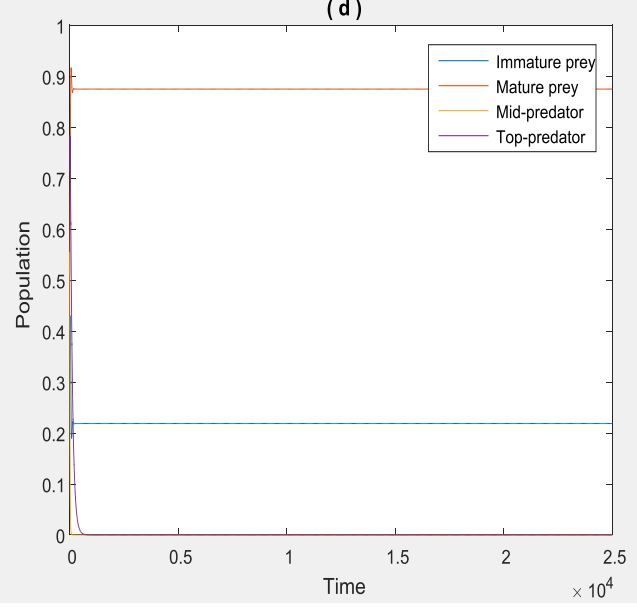

Fig (6.9): - Time series of the solution of system (2) for the data given by(6.1) with different values of $\mathrm{m}$, ( a ): $\mathrm{E}_{3}=(0.28,0.77,0.02,0.06)$ is a asymptotically stable with $\mathrm{m}=0.5$, ( $\left.\mathrm{b}\right)$ : A small periodic attractor with $\mathrm{m}=0.67,(\mathrm{c}): \mathrm{E}_{2}=(0.22,0.86,0.02,0)$ is a asymptotically stable with $\mathrm{m}=0.7,(\mathrm{~d}): \mathrm{E}_{1}=(0.21,0.87,0,0)$ is a asymptotically stable with $\mathrm{m}=0.9$.

\section{Conclusions and discussion:-}

In this chapter, we proposed and analyzed an ecological model that described the dynamical behavior of the food chain real system. The model included four non-linear autonomous differential equations that describe the dynamics of four different population, namely first immature prey $\left(X_{1}\right)$, mature prey $\left(X_{2}\right)$, mid-predator $\left(Y_{1}\right)$ and $\left(Y_{2}\right)$ which is represent the top predator. The boundedness of system (2) has been discussed. The existence conditions of all possible equilibrium points are obtain. The local as well as global stability analyses of these points are carried out. Finally, numerical simulation is used to specific the control set of parameters that affect the dynamics of the system and confirm our obtained analytical results. Therefore system (2) has been solved numerically for different sets of initial points and different sets of parameters starting with the hypothetical set of data given by Eq. (6.1) and the following observations are obtained.

1-System ( 2 ) has two types of attractor in Int. $R_{+}^{4}$ either a stable point or a periodic attractor.

2-For the set hypothetical parameters value given in Eq. (6.1), the system (2) approaches asymptotically to globally stable positive point $E_{3}=(0.28,0.77,0.2,0.06)$. Further, with varying one parameter each time, it is observed 
that varying the parameter values, $u_{i}, i=1,2,4$ and 6 do not have any effect on the dynamical behavior of system( 2 ) and the solution of the system still approaches to positive equilibrium point $E_{3}=\left(x^{*}, y^{*}, z^{*}, w^{*}\right)$.

3-As the natural death rate of immature prey $u_{3}$ increasing to 0.89 keeping the rest of parameters as in Eq. (6.1), the solution of system (2) approaches to positive equilibrium point $E_{3}$. However if $0.90 \leq u_{3}<1$, then the top predator will face extinction then the trajectory transferred from positive equilibrium point to the equilibrium point $E_{2}=$ $(\hat{x}, \hat{y}, \hat{z}, 0)$, thus, the $u_{3}=0.9$ parameter is a bifurcation point.

4- As the natural death rate of mature prey $u_{5}$ increasing to 0.40 keeping the rest of parameters as in Eq. (6.1), the solution of system (2) approaches to positive equilibrium $E_{3}$, however if $0.41 \leq u_{5}<0.58$, then the top predator will face extinction then the trajectory transferred from positive equilibrium point to the equilibrium point $E_{2}=$ $(\hat{x}, \hat{y}, \hat{z}, 0)$, further increasing in the range $0.58 \leq u_{5}<0.8$ causes the mid-predator faced extinction in andthen the trajectory transferred from the free top predator equilibrium point $E_{2}=(\hat{x}, \hat{y}, \hat{z}, 0)$, to the free predators equilibrium point $\mathrm{E}_{1}=(\bar{x}, \bar{y}, 0,0)$, then more increasing of this parameter in the range $0.8 \leq u_{5}<1$ causes extinction in all species and then the trajectory transferred from equilibrium point $\mathrm{E}_{1}=(\bar{x}, \bar{y}, 0,0)$,to the vanishing equilibrium point $\mathrm{E}_{0}=(0,0,0,0)$, thus, the $u_{5}$ parameter when $u_{5}=0.41, u_{5}=0.58$ and $u_{5}=0.8$ is a bifurcation point.

5- As theparameter $u_{7}$ which represents the conversion rate from the mature prey to the mid-predator decreasing to 0.15 keeping the rest of parameters as in Eq.(6.1), the solution of system ( 2 ) approaches to the positive free predators equilibrium point $\mathrm{E}_{1}=(\bar{x}, \bar{y}, 0,0)$, while for the $u_{7}=0.15$, then the trajectory transferred from the free predators equilibrium point $\mathrm{E}_{1}=(\bar{x}, \bar{y}, 0,0)$ to $\mathrm{E}_{2}=(\hat{x}, \hat{y}, \hat{z}, 0)$, which means revival of the mid-predator population, then increasing this parameter in the range $0.15 \leq u_{7}<0.21$ leads revival of the top-predator and the trajectory approaches asymptotically to a periodic dynamics in Int. $R_{+}^{4}$, for more increasing in the range $0.21 \leq$ $u_{7}<0.5$, the trajectory will transferred asymptotically from a periodic dynamics in Int. $R_{+}^{4}$ and then approaches asymptotically stable to a positive equilibrium point $E_{3}\left(x^{*}, y^{*}, z^{*}, w^{*}\right)$, thus, the parameter $u_{7}$ when $u_{7}=0.15$ and $u_{7}=0.21$ is a bifurcation point.

6-As the natural death rate of the mid predator $u_{8}$ increasing to 0.15 keeping the rest of parameters as in Eq.(6.1), the solution of system ( 2 ) approaches to the positive equilibrium point $E_{3}$, further increasing in the range $0.16 \leq$ $u_{8}<0.18$ causes the top-predator faced extinction and the trajectory transferred from the positive equilibrium point $E_{3}$ to the free top predator equilibrium point $E_{2}=(\hat{x}, \hat{y}, \hat{z}, 0)$, but for $0.18 \leq u_{8}<1$ causes the mid -predator faced extinction and the trajectory transferred from $\mathrm{E}_{2}=(\hat{x}, \hat{y}, \hat{z}, 0)$ to $\mathrm{E}_{1}=(\bar{x}, \bar{y}, 0,0)$, thus, the parameter $u_{8}$ when $u_{8}=0.16$ and $u_{8}=0.18$ is a bifurcation point.

7-As the predation rate parameter of the top-predator upon the mid-predator $u_{9}$ increasing to 0.14 keeping the rest of parameters as in Eq.(6.1), the solution of system ( 2 ) approaches the free top predator equilibrium point $E_{2}=$ $(\hat{x}, \hat{y}, \hat{z}, 0)$, while for $0.15 \leq u_{9}<1$ the top-predator population revives and then the trajectory transferred from the point $E_{2}=(\hat{x}, \hat{y}, \hat{z}, 0)$ to the positive equilibrium point $E_{3}$, thus, the parameter $u_{9}=0.15$ is a bifurcation point.

8- As the natural death rateof the top-predator parameter $u_{10}$ increasing in the range $0.1 \leq u_{10}<0.35$ keeping the rest of parameters as in Eq. (6.1) the solutionof system (2)approaches asymptotically to a positive equilibrium point $E_{3}$, while increasing this parameter in the range $0.35 \leq u_{10}<1$ causes extinction of the top-predator population and then the trajectory transferred from positive equilibrium point to $E_{2}=(\hat{x}, \hat{y}, \hat{z}, 0)$, thus, the parameter $u_{10}=0.35$ is a bifurcation point.

9- As the number of prey inside the refuge parameter $m$ varying in the range $0.01 \leq m<0.63$ and keeping the rest of parameters values as data given in Eq. ( 6.1), the solution of system ( 2 ) approachesasymptotically to the positive equilibrium point $E_{3}$, while increasing this parameter in the range $0.63 \leq m<0.69$ leads that the trajectory approaches asymptotically to a periodic dynamics in Int. $R_{+}^{4}$, while increasing this parameter for $0.69 \leq \mathrm{m}<0.71$ causes extinction of the top-predator population and restore the stability andthen the trajectory transferred asymptotically from a periodic dynamics in Int. $R_{+}^{4}$ to the stablefree top-predator equilibrium point $E_{2}=$ $(\hat{x}, \hat{y}, \hat{z}, 0)$, then more increasing of this parameter for $0.71 \leq m$ causes extinction of the mid-predator population and the trajectory transferred from $\mathrm{E}_{2}=(\hat{x}, \hat{y}, \hat{z}, 0)$, to $\mathrm{E}_{1}=(\bar{x}, \bar{y}, 0,0)$, thus, the $m$ parameter when $m=0.63, m$ $=0.69$ and $m=0.71$ is a bifurcation point. 


\section{References:-}

1. Aiello, W. and Freedman, H., (1990) :A time-delay model of single-species growth with stage structure, Math. Biosci.,101(2),139-153,

2. Aiello, W., Freedman, H. and Wu, J., (1992):Analysis of a model representing stage-structured population growth with state-dependent time delay, SIAM J. Appl. Math.,855-869.

3. Azhar, A. M. and Noor, H. A.,April [2016]: Stability analysis of stage structured prey-predator model incorporating a preyrefuge Global Journal Of Engineering Science And Researches, Majeed,3(4): ISSN 2348 8034.

4. Azhar, M. A. and Inaam, I. Sh.,(2016): the Dynamics of an Eco- Epidemiological Model with (SI), (SIS) Epidemic Disease in Prey, Gen. Math. Notes, Vol.34, No. 2, pp.52- 74.

5. Baurmann, M., Gross, T., Feudel, U.,(2007): Instabilities in spatially extended predator -prey systems: spatiotemporal patterns in the neighborhood of Turing-Hopf bifurcations. J. Theor. Biol. 245, 220-229.

6. Berryman, A. A.,(1992): The orgins and evolution of predator-prey theory Ecology, 73 (5) pp. 1530-1535.

7. Catfish Hunt Pigeons: Fish Catch Birds On Land In Display Of Adaptive Behavior (VIDEO), 12/08/2012 04:22 pm ET | Updated Dec 08, 2012.

8. Chen L, Chen F, Chen L (2010) Qualitative analysis of a predator prey model with Holling type II functional response incorporating a constant prey refuge.Nonlinear Anal Real World Apply 11:246-252.

9. Collings, J.B.,(1995): Bifurcation and stability analysis of a temperature dependent mite predator-prey interaction model incorporating a prey refuge. Bull. Math. Biol. 57, 63-76.

10. Florida Museum of Natural History: Prionaceglauca.

11. Freedman, H.I.,(1980): Deterministic Mathematical Model in Population Ecology. Marcel Dekker, New York.

12. González-Olivars, E., Ramos-Jiliberto, R.,(2003): Dynamics consequences of prey refuges in a simple model system: more prey, few predators and enhanced stability. Ecol. Model. 166, 135-146.

13. Ives, A.R., Dobson, A.P.,(1987): Antipredator behavior and the population dynamics of simple predator-prey systems. Am. Nat. 130, 431-447.

14. Kar.TK (2005) Stability analysis of a prey-predator model incorporating a prey refuge. Communications in Nonlinear Scienceand Numerical Simulation 10 (2005) 681-691.

15. Lotka, A. M.(1925), the Elements of Physical Biology, Williams and Wilkins, Baltimore.

16. Ma Z, Li W, Zhao Y, Wang W, Zhang H, Li Z (2009) Effects of prey refuges on a predator-prey model with a class of function responses: the role of refuges. Math Biosci 218:73-79.

17. May, R.M.,(1974): Stability and Complexity in Model Ecosystems. Princeton University Press, Princeton

18. Meinhardt,H.,(1995):Growthandpatterning-dynamicsofstripeformation.Nature376,722-723

19. Naji, R. K. and Mustafa, A. N.,(2012):Journal of Applied Mathematics Volume (2012), Article ID 852631, 24 pages.

20. Pal, D., Mahapatra, GS.,Samanta, GP (2012) A proportional harvesting dynamical model with fuzzy intrinsic growth rate and harvesting quantity. Pacific Asian J Math 6:199-213.

21. Pal, D., Mahapatra, GS.,Samanta, GP (2013) Optimal harvesting of prey-predator system with interval biological parameters: a bioeconomic model. Math Biosci 241:181-187.

22. Pal, D., Mahapatra, GS.,Samanta, GP (2013) Quota harvesting model for a single species population under fuzziness. Int J Math Sci 12:33-46.

23. Pal, D., Mahapatra,GS., Samanta, GP (2014) a bioeconomicmodelingof two prey and one-predator fishery model with optimalharvesting policy through hybridization approach. Appl Math Comput 242:748-763.

24. Pal, D., Mahapatra, GS.,Samanta, GP (2015) Stability and bionomic analysis of fuzzy parameter based preypredator harvesting model using UFM. Nonlinear Dyn 79:1939-1955.

25. Samanta, G.P., 15 (1) (2010), Analysis of a delay nonautonous predator-prey system with disease in the prey Nonlinear Anal.: Modell. Contr., pp. 97-108.

26. Sun,G.,Jin,Z.,Liu,Q.X.,Li,L.:Patternformationina spatialS-Imodel with nonlinearincidencerates.J.Stat.Mech.P11011(2007).

27. Sun,G.Q.,Jin,Z.,Liu,Q.X.,Li,L.,(2008),:Chaosinducedbybreakupofwavesinaspatialepidemicmodelwithnonlinearincidencerate.J.Stat.Mech.P08011.

28. Voltera, V., 2 (1926), :Variazioni e fluttuazioni del numero di individui in specie animaliconviventi, Mem. Accd.Lincei., pp. 1-113.

29. Zahraa, J. K. ,Azhar, A. M. and Naji, R. K.,(2015): Stability analysis of two predator-one stage-structured prey model incorporating a prey refuge, IOSR Journal of Mathematics, vollum 11 No.1. 